\title{
Can Winds Driven by Active Galactic Nuclei Account for the Extragalactic Gamma-Ray and Neutrino Backgrounds?
}

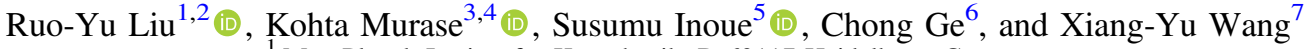 \\ ${ }^{1}$ Max-Planck-Institut für Kernphysik, D-69117 Heidelberg, Germany \\ ${ }^{2}$ Deutsches Elektronen-Synchrotron (DESY), Platanenallee 6, D-15738 Zeuthen, Germany; ruoyu.liu@ desy.de \\ ${ }^{3}$ Department of Physics; Department of Astronomy \& Astrophysics; Center for Particle and Gravitational Astrophysics, The Pennsylvania State University, \\ University Park, Pennsylvania 16802, USA \\ ${ }^{4}$ Yukawa Institute for Theoretical Physics, Kyoto, Kyoto, 606-8502, Japan \\ 5 Astrophysical Big Bang Laboratory, RIKEN, 2-1 Hirosawa, Wako 351-0198 Japan \\ ${ }^{6}$ Department of Physics, University of Alabama in Huntsville, Huntsville, AL 35899, USA \\ ${ }^{7}$ School of Astronomy and Space Science, Nanjing University, Nanjing 210093, People's Republic of China \\ Received 2017 December 24; revised 2018 March 25; accepted 2018 March 26; published 2018 April 26
}

\begin{abstract}
Various observations are revealing the widespread occurrence of fast and powerful winds in active galactic nuclei (AGNs) that are distinct from relativistic jets, likely launched from accretion disks and interacting strongly with the gas of their host galaxies. During the interaction, strong shocks are expected to form that can accelerate nonthermal particles to high energies. Such winds have been suggested to be responsible for a large fraction of the observed extragalactic gamma-ray background (EGB) and the diffuse neutrino background, via the decay of neutral and charged pions generated in inelastic $p p$ collisions between protons accelerated by the forward shock and the ambient gas. However, previous studies did not properly account for processes such as adiabatic losses that may reduce the gamma-ray and neutrino fluxes significantly. We evaluate the production of gamma rays and neutrinos by AGNdriven winds in detail by modeling their hydrodynamic and thermal evolution, including the effects of their twotemperature structure. We find that they can only account for less than $\sim 30 \%$ of the EGB flux, as otherwise the model would violate the independent upper limit derived from the diffuse isotropic gamma-ray background. If the neutrino spectral index is steep with $\Gamma \gtrsim 2.2$, a severe tension with the isotropic gamma-ray background would arise as long as the winds contribute more than $20 \%$ of the IceCube neutrino flux in the $10-100 \mathrm{TeV}$ range. At energies $\gtrsim 100 \mathrm{TeV}$, we find that the IceCube neutrino flux may still be accountable by AGN-driven winds if the spectral index is as small as $\Gamma \sim 2.0-2.1$.
\end{abstract}

Key words: galaxies: active - gamma rays: diffuse background - ISM: jets and outflows - neutrinos

\section{Introduction}

Powerful, highly collimated jets of predominantly nonthermal plasma with ultrarelativistic outflow velocities are seen to be produced in less than $10 \%$ of all active galactic nuclei (AGNs; e.g., Peterson 1997). On the other hand, there is widespread evidence that AGNs can more commonly eject moderately collimated winds of thermal plasma, with outflow velocities from a few thousand kilometers per second up to mildly relativistic values of $\sim 0.3 c$ (where $c$ is the speed of light), primarily observed as blueshifted absorption features due to ionized metals at UV and X-ray energies in Seyfert galaxies and quasars (for reviews, see, e.g., Crenshaw et al. 2003; Veilleux et al. 2005; Fabian 2012; King \& Pounds 2015; Tombesi 2016). The winds are inferred to be generated on subparsec scales, and their estimated kinetic power can reach a fair fraction of the AGN bolometric luminosity. Recent observations of fast and massive outflows of atomic and molecular material in AGNs are likely evidence that such winds propagate to kiloparsec scales and interact strongly with the gas in their host galaxies (e.g., Cicone et al. 2014; Tombesi et al. 2015).

Such AGN-driven winds may be launched from accretion disks by a variety of mechanisms involving thermal, radiative, and magnetic processes (Crenshaw et al. 2003; Ohsuga \& Mineshige 2014). They may be the primary agents by which supermassive black holes (SMBHs) provide mechanical and/or thermal feedback onto their host galaxies, potentially leading to the observed black hole scaling relations and/or the quenching of star formation in massive galaxies (for reviews, see, e.g., Fabian 2012; Kormendy \& Ho 2013; Heckman \& Best 2014; King \& Pounds 2015). Some recent theoretical studies have addressed the interaction of AGN-driven winds with the ambient gas in the host galaxy and/or halo, with particular attention to the physics of the resulting shocks and its observational consequences (Jiang et al. 2010; FaucherGiguère \& Quataert 2012; Nims et al. 2015; Wang \& Loeb 2015).

The extragalactic gamma-ray background (EGB) has been measured in the GeV-TeV range by the Fermi Large Area Telescope (Fermi-LAT; Ackermann et al. 2015), and the diffuse neutrino intensity at $\gtrsim 10 \mathrm{TeV}$ energies has been observed by the IceCube Neutrino Observatory (e.g., Aartsen et al. 2013, 2015; IceCube Collaboration 2013; Halzen 2017). Astrophysical gamma rays and neutrinos can be produced via the decay of neutral $\left(\pi^{0} \rightarrow \gamma \gamma\right)$ and charged pions $\left(\pi^{ \pm} \rightarrow e^{ \pm} \nu_{\mu} \nu_{e}\right)$, and one of the main meson production mechanisms is the hadronuclear reaction (or inelastic $p p$ collision) between high-energy cosmic rays and cold nucleons in the ambient medium. It has been suggested that when the AGN-driven wind expands into the interstellar medium (ISM) of the host galaxy, cosmic rays with nuclear charge number $Z$ are accelerated up to $\sim Z \times\left(10^{16}-10^{17}\right) \mathrm{eV}$ by the forward shock (Tamborra et al. 2014; Wang \& Loeb 2016a, 2016b, hereafter WLI and WLII; Lamastra et al. 2017). Tamborra et al. (2014; see also Murase et al. 2014) pointed out that the 
contributions of starburst galaxies coexisting with AGNs are necessary for star-forming galaxies to significantly contribute to the diffuse neutrino and gamma-ray backgrounds, and they suggested the possibility of AGN-driven winds as one of the cosmic-ray accelerators. However, realistically, the theoretical gamma-ray and neutrino fluxes highly depend on the model parameters, such as the shock velocity evolution and the density of the ambient medium, which determines the interaction efficiency, as studied in WLI, WLII, and Lamastra et al. (2017). Actually, as we will show in this work, the total diffuse neutrino background and EGB cannot be simultaneously explained by this model, once considering the constraint from the so-called isotropic gamma-ray background (IGRB), which is obtained by subtracting the emission of resolved extragalactic point sources from the EGB (Ackermann et al. 2015).

In this work, we evaluate the gamma-ray and neutrino emission from AGN-driven winds in more detail compared to previous studies. We take into account several effects that had not been properly accounted for, such as the two-temperature structure of the wind and the adiabatic cooling of accelerated protons. The resulting diffuse gamma-ray and neutrino fluxes are reduced, by which we can avoid the problem of overshooting the IGRB. The paper is structured as follows: the dynamical evolution of the wind is studied in Section 2; gamma-ray and neutrino production by an individual source is calculated in Section 3; we obtain the diffuse gamma-ray and neutrino flux from the sources throughout the universe and compare with the results in the previous literature in Section 4; in Sections 5 and 6, we discuss various implications of our results; and the summary is given in Section 7.

\section{Dynamics of AGN-driven Winds}

Following WLI, WLII, and Lamastra et al. (2017), we adopt the 1D model and assume the spherical symmetry for the wind and the ambient gas. The physical picture is similar to that of the stellar-wind bubble (Castor et al. 1975) but in different scales. Let us denote the radius of the forward shock that expands into the ambient medium by $R_{\mathrm{s}}$, and the radius of the reverse shock that decelerates the wind by $R_{\mathrm{rs}}$. Together with a contact discontinuity at radius $R_{\mathrm{cd}}$ that separates the two shocks, this dynamical system is divided into four distinct zones. Outward, they are (a) the cold fast AGN wind moving with the bulk velocity $v_{\mathrm{w}}$, (b) the hot shocked winds, (c) the shocked ambient gases, and (d) the ambient gas, which is assumed to consist of pure hydrogen atoms for simplicity. A schematic diagram that illustrates the outflow structure is shown in Figure 1. Following the treatment in the previous literature (Weaver et al. 1977; Faucher-Giguère \& Quataert 2012; Wang \& Loeb 2015), we consider the so-called thin-shell approximation for region $\mathrm{c}$, which assumes negligible thickness of the shocked ambient gases (i.e., $R_{\mathrm{cd}} \simeq R_{S}$ ) and that all the shocked gases move with the same velocity $v_{\mathrm{s}}{ }^{8}$ In region $\mathrm{b}$ or the region of shocked AGN wind, we consider a steady flow of a homogeneous density $n_{\mathrm{sw}}$ and temperature $T_{\mathrm{sw}}$, which results in a homogeneous thermal pressure $P_{\mathrm{sw}}$ in the region at any given time. The condition of mass conservation then gives a constant value of $R^{2} v_{\mathrm{sw}}$ from $R_{\mathrm{rs}}$ to $R_{s}$, where $R$ is the distance

\footnotetext{
8 The forward-shock speed should be about 4/3 times the downstream speed when the Mach number is large. But they are essentially the same under the thin-shell approximation.
}

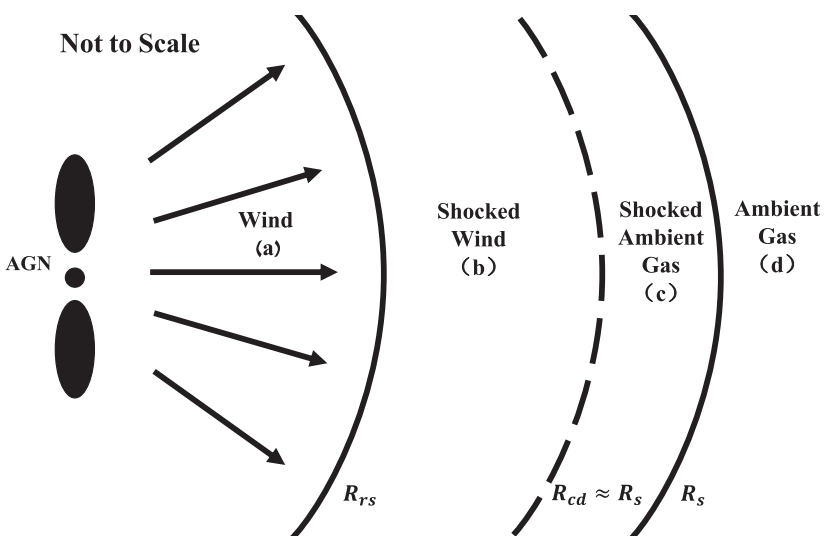

Figure 1. Schematic diagram for the structure of the AGN wind-shock system. Spherical symmetry is assumed for the system. See the text for detailed descriptions.

to the AGN at the galactic center and $v_{\mathrm{sw}}$ is the velocity of the shocked wind. At $R=R_{s}$, the shocked wind should move at the same velocity as the shocked gas, so we have the boundary condition, $v_{\mathrm{sw}}\left(R_{\mathrm{S}}\right)=v_{\mathrm{s}}$. Let's further denote the velocity of the shocked wind just behind the reverse shock by $v_{\mathrm{sw}}\left(R_{\mathrm{rs}}\right)=v_{\mathrm{sw}}^{\prime}$, and then we have $v_{\mathrm{sw}}^{\prime}=\left(R_{s} / R_{\mathrm{rs}}\right)^{2} v_{s}$. We note that the velocity of the shocked wind just behind the reverse shock is not equal to that of the reverse shock $v_{\mathrm{rs}}$. But we can find the relation between them by the Rankine-Hugoniot jump relation, i.e.,

$$
v_{w}-v_{\mathrm{rs}}=4\left(v_{\mathrm{sw}}^{\prime}-v_{\mathrm{rs}}\right) .
$$

Besides, this condition gives the proton and electron temperatures in shocked wind immediately behind the shock by

$$
\begin{aligned}
& T_{p, \mathrm{sw}}=\frac{3}{16} \frac{m_{p}}{k}\left(v_{\mathrm{w}}-v_{\mathrm{rs}}\right)^{2}, \\
& T_{e, \mathrm{sw}}=\frac{3}{16} \frac{m_{e}}{k}\left(v_{\mathrm{w}}-v_{\mathrm{rs}}\right)^{2},
\end{aligned}
$$

where $m_{p}$ and $m_{e}$ are the mass of a proton and an electron, respectively. We consider the minimal electron heating case, protons receive the majority of the shock heat (FaucherGiguère \& Quataert 2012), and the thermal pressure of the shocked wind can then be found by

$$
\begin{aligned}
P_{p, \mathrm{sw}} & =n_{\mathrm{sw}} k T_{\mathrm{sw}}=\frac{3}{16} n_{\mathrm{sw}} m_{p}\left(v_{\mathrm{w}}-v_{\mathrm{rs}}\right)^{2} \\
& =\frac{1}{3} n_{\mathrm{sw}} m_{p}\left(v_{\mathrm{w}}-v_{\mathrm{sw}}^{\prime}\right)^{2}, \\
P_{e, \mathrm{sw}} & =n_{\mathrm{sw}} k T_{\mathrm{sw}}=\frac{3}{16} n_{\mathrm{sw}} m_{e}\left(v_{\mathrm{w}}-v_{\mathrm{rs}}\right)^{2} \\
& =\frac{1}{3} n_{\mathrm{sw}} m_{e}\left(v_{\mathrm{w}}-v_{\mathrm{sw}}^{\prime}\right)^{2},
\end{aligned}
$$

and the total thermal pressure is $P_{\mathrm{sw}}=P_{p, \mathrm{sw}}+P_{e, \mathrm{sw}}$. In the above expressions, $n_{\mathrm{sw}}=4 n_{\mathrm{w}}=\dot{M}_{\mathrm{w}} / \pi R_{\mathrm{rs}}^{2} m_{p} v_{\mathrm{w}}$ is the density of both protons and electrons in the shocked wind, where $n_{\mathrm{w}}$ is the density of the unshocked wind and $\dot{M}_{\mathrm{w}}=2 L_{\mathrm{w}, \mathrm{k}} / v_{\mathrm{w}}^{2}$ is the mass injection rate of the wind, with $L_{\mathrm{w}, \mathrm{k}}$ being the kinetic luminosity of the wind. We assume $L_{\mathrm{w}, \mathrm{k}}$ to be $5 \%$ of the bolometric luminosity of the AGN $L_{b}$ following WLII, keeping constant before the AGN quenches. Note that the sound speed in the shocked wind region is $\sim \sqrt{P_{\mathrm{sw}} / \rho_{\mathrm{sw}}} \simeq v_{\mathrm{w}}-v_{\mathrm{sw}}^{\prime}$, which is 
generally larger than $v_{s}$. Thus, the sound-crossing time is shorter than the dynamical timescale, and this validates the previous assumptions of a homogeneous density, temperature, and thermal pressure in this region.

The total thermal energy in the shocked wind region, or region $\mathrm{b}$, is then $E_{\mathrm{t}, \mathrm{sw}}=\frac{3}{2} P_{\mathrm{sw}} V_{\mathrm{sw}}=\frac{1}{2} M_{\mathrm{sw}}\left(v_{\mathrm{w}}-v_{\mathrm{sw}}^{\prime}\right)^{2}$, with $V_{\mathrm{sw}}=\frac{4}{3} \pi\left(R_{s}^{3}-R_{\mathrm{rs}}^{3}\right)$ being the volume of the shocked wind. Similarly, we have that the total thermal energy in the shocked gas (region c) is $E_{\mathrm{t}, \mathrm{sg}}=\frac{1}{2} M_{\mathrm{sg}} v_{s}^{2} . M_{\mathrm{sw}} \simeq \dot{M}_{\mathrm{w}}\left(R_{s} / v_{s}-R_{\mathrm{rs}} / v_{\mathrm{w}}\right)$ is the mass of the shocked wind at time $t \sim R_{s} / v_{s}$, and $M_{\mathrm{sg}}=$ $4 \pi \int_{0}^{R_{s}} r^{2} \rho_{g}(r) d r$ is the total mass of the ambient gas swept up by the forward shock, with $\rho_{g}$ being the density profile of the gas. WLI and WLII adopted a broken power-law (BPL) distribution for $\rho_{g}$, i.e., $\rho_{g} \propto R^{-2}$ if $R<R_{\text {disk }}$ and $\rho_{g} \propto R^{-3.95}$ if $R>R_{\text {disk }}$ for a disk component and halo component of the gas, respectively, where the disk radius is related to the virial radius of the galaxy by $R_{\text {disk }}=0.04 R_{\text {vir. }}$. However, such a setup will lead to an extremely high gas density at small radius. Usually, the average number density of the ISM is approximately constant in the disk of a galaxy and not significantly larger than $\sim 10^{4} \mathrm{~cm}^{-3}$ even for the starburst region of ULIRGs, which are rich in gas (such as the core of Arp 220; Downes \& Solomon 1998; Peng et al. 2016). Thus, we add a flat core in the density profile, i.e., $\rho_{0}=1000 m_{p}\left(L_{b} / 10^{45} \mathrm{ergs}^{-1}\right)^{1 / 3} \mathrm{~cm}^{-3}$, for the inner $100 \mathrm{pc}$. On the other hand, there are intergalactic medium surrounding galaxies, so the gas density may not keep decreasing at large radius, and we assume the gas density beyond the virial radius to be $\rho_{g}\left(R>R_{\mathrm{vir}}\right)=\rho_{g}\left(R=R_{\mathrm{vir}}\right)$. In summary, the adopted gas density profile in our calculation is

$$
\rho_{g}=\left\{\begin{array}{l}
\frac{\rho_{0}}{1+(R / 100 \mathrm{pc})^{2}} \mathrm{~cm}^{-3}, \quad R<R_{\text {disk }}, \\
\frac{\rho_{0}}{1+\left(R_{\text {disk }} / 100 \mathrm{pc}\right)^{2}}\left(\frac{R}{R_{\text {disk }}}\right)^{-3.95} \mathrm{~cm}^{-3}, \quad R_{\text {disk }}<R<R_{\text {vir. }} . \\
\frac{\rho_{0}}{1+\left(R_{\text {disk }} / 100 \mathrm{pc}\right)^{2}}\left(\frac{R_{\text {vir }}}{R_{\text {disk }}}\right)^{-3.95} \mathrm{~cm}^{-3}, \quad R>R_{\text {vir }}
\end{array}\right.
$$

The virial radius of a galaxy of a dark matter halo with mass $M_{h}$ can be given by

$$
\begin{aligned}
R_{\mathrm{vir}}= & 170 h^{-2 / 3}\left[\left(\Omega_{M}(1+z)^{3}+\Omega_{\Lambda}\right)\right. \\
& \left.\times \Delta_{c} / 18 \pi^{2}(1+z)^{3}\right]^{-1 / 3} \\
& \times\left(\frac{M_{\mathrm{h}}}{10^{12} M_{\odot}}\right)^{1 / 3}(1+z)^{-1} \mathrm{kpc},
\end{aligned}
$$

where $\Delta_{c}$ is typically defined as the ratio of the average gas density within the virial radius of the galaxy to the critical density, and its value is $\simeq 18 \pi^{2}$ for a flat universe.

We then can calculate the dynamic evolution of the shocked ambient gas, whose motion is governed by

$$
\frac{d}{d t}\left(M_{\mathrm{sg}} v_{\mathrm{sw}}\right)=-\frac{G M_{G}\left(<R_{s}\right) M_{\mathrm{sg}}}{R_{s}^{2}}+4 \pi R_{s}^{2}\left(P_{\mathrm{sw}}-P_{g}\right),
$$

where the first term on the right-hand side of the equation considers the gravity on the expanding shell, exerted by the total gravitational mass within $R_{s}$, including the $\mathrm{SMBH}$, the dark matter, and the self-gravity of the shell of the swept-up gas itself, i.e., $M_{G}\left(<R_{s}\right)=M_{\mathrm{BH}}+M_{\mathrm{DM}}\left(<R_{s}\right)+M_{\mathrm{sg}}\left(<R_{s}\right) / 2 . M_{\mathrm{DM}}\left(<R_{s}\right)$ is calculated based on the NFW profile (Navarro et al. 1996) and the total mass of the dark matter halo $M_{h}$. The values of $M_{h}$ and $M_{\mathrm{BH}}$ are related to the AGN's bolometric luminosity $L_{b}$, which are referred to the treatment by WLII (see also Appendix A.1 for details). $P_{g}$ is the thermal pressure of the unshocked ambient gas, which tends to resist the expansion of the shell. The pressure is related to the gas density and temperature by $P_{g}=n_{g} k T_{g}$. Here, $T_{g}$ can be found by the hydrostatic equilibrium of the gas in the galaxy:

$$
\frac{d T_{g}}{d R}=\frac{G M_{G} m_{p}}{k R^{2}}-\frac{T_{g}}{n_{g}} \frac{d n_{g}}{d R} .
$$

Note that at small radii the gases may not be in the hydrostatic equilibrium, since the density of the gas is so high that the cooling time is short, leading to a cooling inflow that feeds the activity of the SMBH and the star formation. So the temperature of the gases in the inner galaxy is probably lower than that estimated from the hydrostatic equilibrium. However, such an overestimation of the gas temperature will not have a significant effect on the evolution of the shocked gases, because the deceleration of the shocked gas in the inner galaxy is mainly caused by the increasing mass of the swept-up shell. On the other hand, the swept-up shell is pushed forward by the thermal pressure of the shocked wind $P_{\mathrm{sw}}$. As we mentioned earlier, it is related to the total thermal pressure in the shocked wind by

$$
E_{\mathrm{t}, \mathrm{sw}}=\frac{3}{2} P_{\mathrm{sw}} V_{\mathrm{sw}}=2 \pi P_{\mathrm{sw}}\left(R_{s}^{3}-R_{\mathrm{sw}}^{3}\right),
$$

while the changing rate of the thermal energy is generally determined by the energy injection from the wind, the work done on the swept-up shell, and the radiative cooling of the shocked wind. Considering the two-temperature effect, protons and electrons may have different temperatures and undergo different energy-loss processes. Denoting the thermal energies for protons and electrons by $E_{p, \mathrm{sw}}$ and $E_{e, \mathrm{sw}}$, respectively (with $\left.E_{\mathrm{t}, \mathrm{sw}}=E_{p, \mathrm{sw}}+E_{e, \mathrm{sw}}\right)$, we have

$$
\frac{d E_{p, \mathrm{sw}}}{d t}=\frac{1}{2} \dot{M}_{\mathrm{w}}\left(1-\frac{v_{\mathrm{rs}}}{v_{\mathrm{w}}}\right)\left(v_{\mathrm{w}}^{2}-v_{\mathrm{sw}}^{\prime 2}\right)-4 \pi R_{s}^{2} P_{p, \mathrm{sw}} v_{s}-L_{\mathrm{Cou}}
$$

for protons. The first term on the right-hand side of the equation represents the injection from the wind. Note that the thermal energy injection rate is not the kinetic luminosity of the wind $\frac{1}{2} \dot{M}_{\mathrm{w}} v_{\mathrm{w}}^{2}$. This is because, first, the reverse shock also moves forward at a speed of $d R_{\mathrm{rs}} / d t=v_{\mathrm{rs}}$, and hence only a fraction of $1-v_{\mathrm{rs}} / \nu_{\mathrm{w}}$ of the wind material can inject into the shocked wind region in unit time, and second, a part of the energy, $\frac{1}{2} \dot{M}_{\mathrm{w}} v_{\mathrm{sw}}^{\prime 2}$, goes into the kinetic energy of the shocked wind. The second term represents adiabatic losses due to the expansion or the work done on the swept-up shell. The third term, $L_{\mathrm{Cou}}=\left(E_{p, \mathrm{sw}}-E_{e, \mathrm{sw}}\right) / t_{p e}$, is the Coulomb cooling rate of protons due to collisions with electrons. $t_{p e}$ is the timescale for electrons and protons to achieve equipartition. In principle, protons also suffer from the radiative cooling via Compton scattering, synchrotron radiation, and free-free emission. Since the efficiencies of these cooling processes are very low for 

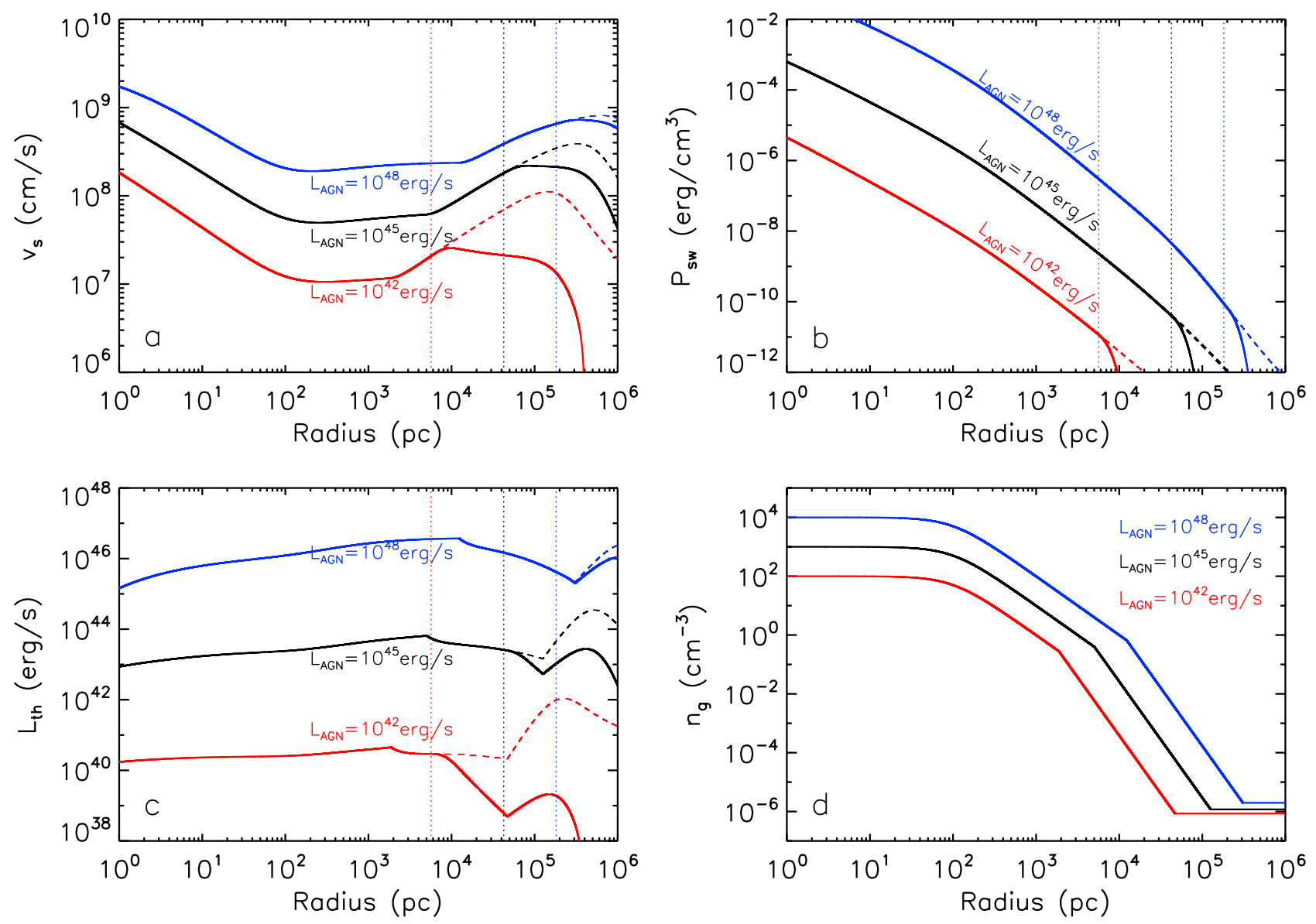

Figure 2. Evolution of forward-shock velocity $\left(v_{s}\right.$; panel (a)), total thermal pressure of the shocked wind $\left(P_{\mathrm{sw}}\right.$; panel (b)), energy injection rate of thermal protons by the forward shock $\left(L_{\mathrm{th}}\right.$; panel (c)), and the density profile of the ambient medium (panel (d)) for AGNs at $z=1$ with $L_{b}=10^{42} \mathrm{erg} \mathrm{s}^{-1}$ (red), $10^{45} \mathrm{erg} \mathrm{s}^{-1}$ (black), and $10^{48} \mathrm{erg} \mathrm{s}^{-1}$ (blue), respectively. The dashed curves are for the evolution without the AGN shutoff. The red, black, and blue vertical dotted lines represent the shock radii after propagating a time of $4 \times 10^{7} \mathrm{yr}$ or the Salpeter time in the case of $L_{b}=10^{42} \mathrm{erg} \mathrm{s}^{-1}, L_{b}=10^{45} \mathrm{erg} \mathrm{s}^{-1}$, and $L_{b}=10^{48} \mathrm{erg} \mathrm{s}^{-1}$, respectively.

protons, we simply neglect them here. But this may not be true for electrons. Thus, we have

$$
\begin{aligned}
\frac{d E_{e, \mathrm{sw}}}{d t}= & \frac{1}{2} \dot{M}_{\mathrm{w}}\left(\frac{m_{e}}{m_{p}}\right)\left(1-\frac{v_{\mathrm{rs}}}{v_{\mathrm{w}}}\right)\left(v_{\mathrm{w}}^{2}-v_{\mathrm{sw}}^{\prime 2}\right) \\
& -4 \pi R_{s}^{2} P_{e, \mathrm{sw}} v_{s}+L_{\mathrm{Cou}}-L_{C}-L_{\mathrm{rad}} .
\end{aligned}
$$

The first two terms on the right-hand side have the same meaning as those in Equation (11). The third term, $L_{\mathrm{Cou}}$, is also exactly the same as that in Equation (11), but with an opposite sign since the Coulomb collision between protons and electrons serves as the heating term for electrons. The fourth term, $L_{\mathrm{C}}$, is the Compton cooling/heating rate through Compton scattering (Sazonov \& Sunyaev 2001; Faucher-Giguère \& Quataert 2012). The last term, $L_{\mathrm{rad}}=E_{e, \mathrm{sw}}\left(1 / t_{\mathrm{ff}}+1 / t_{\mathrm{syn}}\right)$, is the radiative cooling rate due to free-free emission and synchrotron emission, with $t_{\mathrm{ff}}$ and $t_{\mathrm{syn}}$ being the cooling timescales (see Appendix A.2 for details).

Based on Equations (8)-(12), we obtain the evolution of various quantities after we find out the initial condition of those quantities (see Appendix A.3 for details). Figure 2 shows the evolution of forward-shock speed $v_{s}$, the thermal pressure in the shocked wind region $P_{\mathrm{sw}}$, and the energy injection rate of thermalized protons from the forward shock $L_{\mathrm{th}}$, as well as the density profile of ambient gas for reference, for AGNs with
$L_{b}=10^{42}, 10^{45}$, and $10^{48} \mathrm{erg} \mathrm{s}^{-1}$ at $z=1$. In panel (a), dashed curves show the case of a constant wind injection, and we can see three breaks in the curves for $v_{s}$, because of the change in the profile of the gas density. A self-similar analytical solution to $v_{s}$, assuming that half of the kinetic energy injected by the wind constantly goes into kinetic motion of the swept-up shell, reads $v_{s} \propto R^{(\alpha-2) / 3}$ (Faucher-Giguère \& Quataert 2012), with $\alpha$ the power-law index of the density profile. For the employed profile, we have $\alpha=0,2,3.95(\simeq 4)$, and 0 , leading to $v_{s} \propto R^{-2 / 3}, R^{0}, R^{2 / 3}$, and $R^{-2 / 3}$, respectively, for the core region, the disk, the halo, and outside virial radius (dashed curves). Our results do not deviate much from this analytical one, implying that radiation losses are not severe and the flow is "energy driven." The dotted vertical lines in the figures mark the Salpeter time $t_{\text {sal }} \simeq 4 \times 10^{7} \mathrm{yr}$ for a radiative efficiency of 0.1 , which is regarded as the lifetime of the quasars (e.g., Yu \& Tremaine 2002). Beyond the lifetime, we assume that the AGN shuts off and hence the injection of the wind vanishes, as in WLII. We can see that the swept-up shell of shocked gas will not stop immediately, continuing to expand but with a different dynamic evolution quickly after the Salpeter time (solid curves). Without the further energy injection into the region of shock wind, the thermal energy therein depletes quite fast, as can be seen in panel (b), and the forward shock starts to be decelerated after losing the push by the thermal pressure of the shocked wind. Note that given a temperature of gas in the halo of a galaxy of $\sim 10^{6} \mathrm{~K}$, the sound speed is supposed to be a few 
times $10^{6} \mathrm{~cm} \mathrm{~s}^{-1}$. Thus, the forward shock may disappear at late times for low-luminosity AGNs (e.g., $L_{b}=10^{42} \mathrm{erg} \mathrm{s}^{-1}$ ). Later, for the calculation of gamma-ray and neutrino production, we will use the evolution with the quenching of AGNs considered.

The energy injection rate of thermalized protons from the forward shock is given by $L_{\mathrm{th}}=4 \pi R_{s}^{2} u_{\mathrm{sg}, \mathrm{t}} v_{s}$, where $u_{\mathrm{sg}, \mathrm{t}}=$ $\frac{9}{32} \rho_{\mathrm{sg}} v_{s}^{2}$ is the thermal energy density of the shock-compressed gas and $\rho_{\mathrm{sg}}=4 \rho_{\mathrm{g}}$. We assume that a fraction $\epsilon_{\mathrm{nt}}=0.1$ of the thermal energy injected per unit time is converted to nonthermal energy of relativistic protons via the forward shock, i.e., the cosmic-ray luminosity $L_{\mathrm{CR}}=\epsilon_{\mathrm{nt}} L_{\mathrm{th}}$. If we use the analytical solution of the dynamic evolution for estimation, we have $L_{\mathrm{CR}} \propto L_{\mathrm{th}} \propto R^{0}$, and this behavior is more or less consistent with our results as shown in panel (c).

Given the adopted gas density profile, the total gas mass in the host galaxy is about $5 \%-10 \%$ of the total mass (including dark matter), while the cosmic mean baryon fraction is about $16 \%$. This may not be unreasonable given the "missing" baryon problem found in many galaxies (see, e.g., Bregman 2007, and references therein). Actually, although our assumption on the gas density is more conservative than those in the previous literature (WLI, WLII; Lamastra et al. 2017), our employed gas density profile might still be an overestimation of gas content for some AGNs' host galaxies. From Equation (6), we can see that the column density ranges from $N_{\mathrm{H}}=6 \times$ $10^{22} \mathrm{~cm}^{-2}$ for the AGN with the lowest luminosity in our calculation (i.e., $L_{b}=10^{42} \mathrm{erg} \mathrm{s}^{-1}$ ) to $N_{\mathrm{H}}=6 \times 10^{24} \mathrm{~cm}^{-2}$ for the most luminous one $\left(L_{b}=10^{48} \mathrm{erg} \mathrm{s}^{-1}\right)$. However, observationally, a large fraction of AGNs (mainly Seyferts) have a smaller column density than $N_{\mathrm{H}}=6 \times 10^{22} \mathrm{~cm}^{-2}$, at any redshift or luminosity (e.g., Ueda et al. 2003, 2014; La Franca et al. 2005; Tozzi et al. 2006; Lusso et al. 2012). Although there also exists many AGNs with column density larger than $6 \times 10^{24} \mathrm{~cm}^{-2}$, such high column densities are found to be predominantly caused by the parsec-scale dusty torus (e.g., Fukazawa et al. 2011; Goulding et al. 2012; Buchner \& Bauer 2017; Liu et al. 2017), depending on the covering factor and viewing angles. Note that the important quantity relevant to the gamma-ray and neutrino production is the gas content associated with the galactic disk and halo rather than the dusty torus. Thus, the adopted gas density profile may lead to a significant overestimation of the gas column density and the subsequent gamma-ray and neutrino production, although such a high-density environment would be reasonable for AGNs coexisting with starbursts (Tamborra et al. 2014). On the other hand, if one wants to keep the gas fraction similar to the cosmic mean value, one may take a larger value for $\rho_{0}$ or assume a shallower decrease of the density profile in the halo. As a reference, we here also consider the dynamical evolution of the wind bubble in an alternative case in which there is no further break in the density profile of gas in the halo, i.e.,

$$
\rho_{g}=\left\{\begin{array}{l}
\frac{\rho_{0}}{1+(R / 100 \mathrm{pc})^{2}} \mathrm{~cm}^{-3}, \quad R<R_{\mathrm{vir}}, \\
\frac{\rho_{0}}{1+\left(R_{\mathrm{vir}} / 100 \mathrm{pc}\right)^{2}} \mathrm{~cm}^{-3}, \quad R>R_{\mathrm{vir}} .
\end{array}\right.
$$

The results are shown in Figure 3. In this case, the forward shock will undergo a constant deceleration. Note that in this case the gas mass fraction can even reach $\sim 30 \%-60 \%$. Given such an abundant gas content, the effective $p p$ reaction optical depth increases at large radius. It is certainly not a realistic case, but we may regard it as an upper limit, and we can see later in Figure 5 that the resultant gamma-ray or neutrino flux does not increase much compared to the case shown in Figure 2.

\section{Gamma-ray and Neutrino Production}

The spectrum of produced gamma rays and neutrinos depends on the parent protons. In this work, we consider two different forms of the proton spectrum. First, following WLI and WLII, the differential cosmic-ray proton density injected at a radius $R$ is assumed to be a single power law (SPL) with a cutoff at the highest energy, i.e.,

$$
\begin{aligned}
N_{p}^{\mathrm{inj}}\left(E_{p}, R\right) & \equiv \frac{d N}{d E_{p}} \\
& =N_{0}(R)\left(\frac{E_{p}}{100 \mathrm{TeV}}\right)^{-\Gamma_{\mathrm{CR}}} \exp \left(-E_{p} / E_{p, \max }\right),
\end{aligned}
$$

where $\Gamma_{\mathrm{CR}}$ is the power-law index for cosmic rays and $E_{p}$, max is the maximum achievable proton energy in the forward shock.

As another form of the proton spectrum, we introduce a break below $100 \mathrm{TeV}$ where the spectrum becomes flat, i.e., a BPL with a cutoff,

$$
N_{p}^{\mathrm{inj}}\left(E_{p}, R\right)=N_{0}(R) \begin{cases}\left(\frac{E_{p}}{100 \mathrm{TeV}}\right)^{-2}, & E_{p}<100 \mathrm{TeV} \\ \left(\frac{E_{p}}{100 \mathrm{TeV}}\right)^{-\Gamma_{\mathrm{CR}}} & \exp \left(-E_{p} / E_{p, \max }\right) \\ & , E_{p}>100 \mathrm{TeV} .\end{cases}
$$

The BPL spectrum can keep the secondary neutrino spectral index consistent with the observed one (Aartsen et al. 2015), while in the meantime it reduces the amount of cosmic rays at low energy and, consequently, the gamma-ray production below $10 \mathrm{TeV}$, compared to the SPL spectrum with the same $\Gamma_{\mathrm{CR}}$. $E_{\max }$ can be found by equating the shock acceleration timescale $t_{\mathrm{acc}}=20 E_{p} c / 3 e B v_{s}^{2}$ and the minimum between the dynamical timescale $t_{\mathrm{dyn}} \approx R / v_{s}$ and the $p p$ cooling time $t_{p p}=1 /\left(0.5 \sigma_{p p} n_{\mathrm{sg}} c\right)$, where $\sigma_{p p}$ is the cross section for $p p$ interactions and $n_{\mathrm{sg}}(R)=4 n_{g}(R)$ is the number density of the compressed gas by the forward shock. The magnetic field $B=\left[12 \pi \epsilon_{B} n_{\mathrm{sg}}(R) k T_{\mathrm{sg}}(R)\right]^{1 / 2}$, where $\epsilon_{B}$ is the equipartition parameter for the magnetic field, which is fixed at 0.1 at any $R ; k$ is the Boltzmann constant; and $T_{\mathrm{sg}}(R)=3 m_{p} v_{s}(R)^{2} / 16 k$ is the temperature of the shocked ambient gas. We have $E_{p, \max } \simeq 2 \times 10^{17}\left(\frac{\epsilon_{B}}{0.1}\right)^{1 / 2}\left(\frac{n_{g}}{1000 \mathrm{~cm}^{-3}}\right)^{1 / 2}\left(\frac{R_{s}}{100 \mathrm{pc}}\right)\left(\frac{v_{s}}{10^{8} \mathrm{~cm} \mathrm{~s}^{-1}}\right)^{2} \mathrm{eV}$ if the dynamical time is shorter and $E_{p, \max } \simeq 10^{17}\left(\frac{\epsilon_{B}}{0.1}\right)^{1 / 2}\left(\frac{n_{g}}{1000 \mathrm{~cm}^{-3}}\right)^{-1 / 2}\left(\frac{\sigma_{p p}}{50 \mathrm{mb}}\right)^{-1}\left(\frac{v_{s}}{10^{8} \mathrm{~cm} \mathrm{~s}^{-1}}\right)^{3} \mathrm{eV}$ if the $p p$ cooling time is shorter. One can find the timescale of relevant processes in Figure 4 for AGNs with different bolometric luminosities. Note that there should be a pre-factor $>1$ in the expression for the acceleration timescale since the spatial diffusion of accelerated particles can be far from the Bohm limit. This can lead to the maximum proton energy easily dropping below $10-100 \mathrm{PeV}$ and hence creating difficulties in the explanation of $\mathrm{PeV}$ neutrinos, although it does not have a significant influence on the production of $\mathrm{GeV}$ 

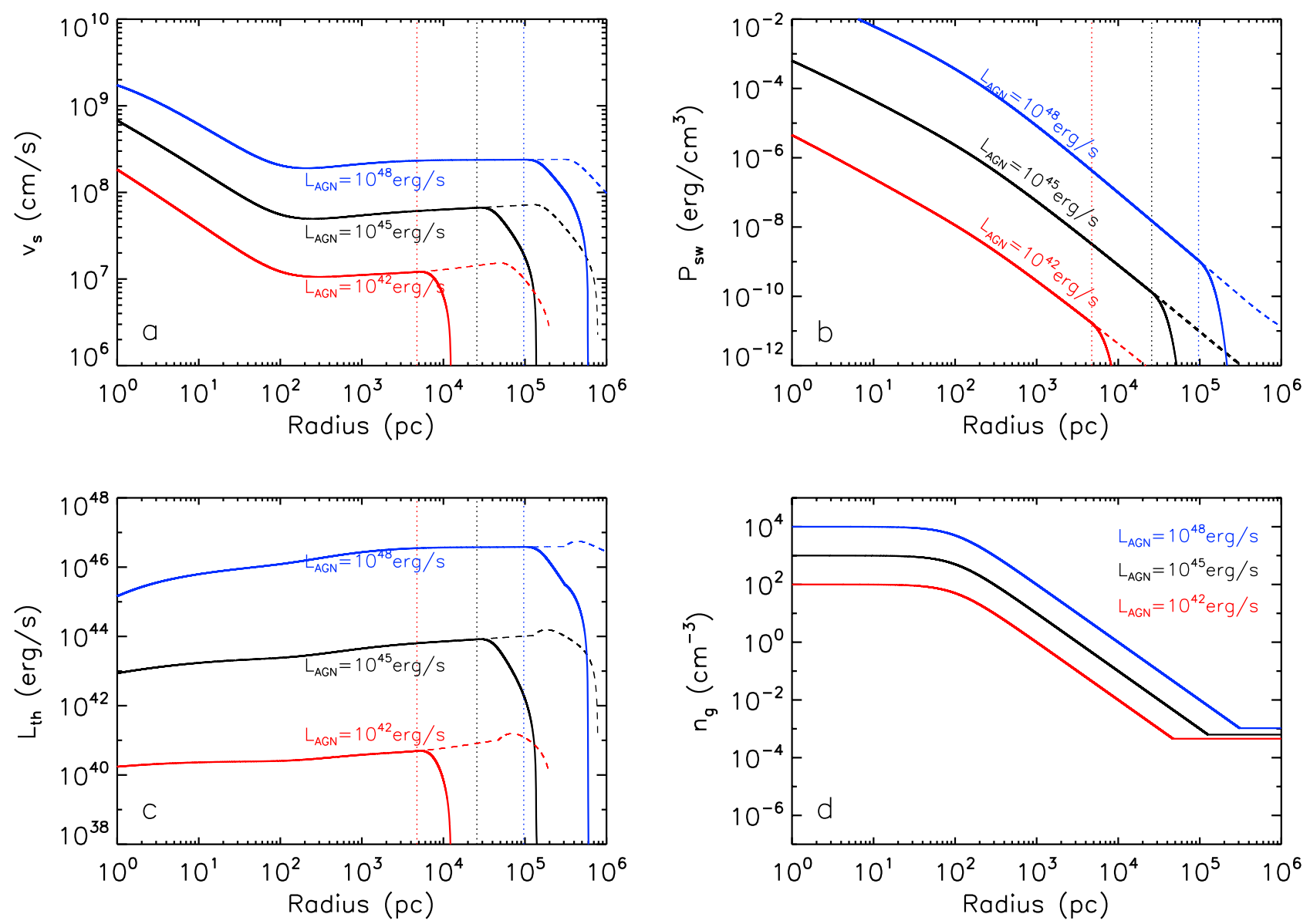

Figure 3. Same as Figure 2, but for the gas density profile without a further break in halo (Equation (13)), as shown in panel (d).

photons. To compare our results with those in the previous literature, we will consider the maximum proton energy obtained in the Bohm limit in the following calculation. The normalization factor $N_{0}(R)$ is found by assuming that a fraction $\epsilon_{\mathrm{nt}}=0.1$ of the newly injected thermal energy in the shocked gas goes into the nonthermal energy of accelerated protons, i.e., $L_{\mathrm{CR}}=\epsilon_{\mathrm{nt}} L_{\mathrm{th}}$ as we mentioned in the previous section. Note that $L_{\mathrm{CR}}=4 \pi R^{2} v_{s} u_{\mathrm{CR}, p}^{\mathrm{inj}}$ with $u_{\mathrm{CR}, p}^{\mathrm{inj}} \equiv \int E_{p} N_{p}^{\mathrm{inj}}\left(E_{p}\right) d E_{p}$ and $L_{\mathrm{th}}=4 \pi R^{2} v_{s} u_{\mathrm{sg}, \mathrm{t}}$ with $u_{\mathrm{sg}, \mathrm{t}}=\frac{3}{2} n_{\mathrm{sg}}(R) k T_{\mathrm{sg}}(R)$. So we have $\int E_{p} N_{p}^{\mathrm{inj}}\left(E_{p}\right) d E_{p}=\frac{3}{2} \epsilon_{\mathrm{nt}} n_{\mathrm{sg}}(R) k T_{\mathrm{sg}}(R)$.

We assume that most accelerated protons are well confined in the downstream of the forward shock (or region b) and interact with gas therein during the advection, since the Larmor radius of a proton reads

$$
\begin{aligned}
r_{L} \simeq & 10^{15}\left(\frac{E_{p}}{10^{15} \mathrm{eV}}\right)\left(\frac{\epsilon_{B}}{0.1}\right)^{-1 / 2}\left(\frac{n_{g}}{1000 \mathrm{~cm}^{-3}}\right)^{-1 / 2} \\
& \times\left(\frac{v_{s}}{10^{8} \mathrm{~cm} \mathrm{~s}^{-1}}\right)^{-1} \mathrm{~cm},
\end{aligned}
$$

which is much smaller than the shock radius. The spectra of secondaries in $p p$ collisions are calculated based on the semianalytical method developed by Kelner et al. (2006). In the optically thin limit, the spectral index of gamma rays and neutrinos, $\Gamma$, is roughly estimated to be $\Gamma \approx \Gamma_{\mathrm{CR}}-0.1$. However, in the calorimetric limit, we expect $\Gamma \approx \Gamma_{\mathrm{CR}}$. For clarity of expression, let's define an operator $\mathcal{F}_{\gamma}$ that can obtain the gamma-ray emissivity by $N_{\gamma}\left(E_{\gamma}\right)=\mathcal{F}_{\gamma}\left\{N_{p}\left(E_{p}\right), n_{\text {sg }}\right\}$ (basically it is the same as for neutrino emissivity; we just need to use another operator $\mathcal{F}_{\nu}$ ), with

$$
\mathcal{F}_{i}\left(E_{i}\right)=c n_{\mathrm{sg}} \int_{E_{i}}^{\infty} \sigma_{p p} d N_{p} d E_{p}\left(E_{p}\right) F_{i}\left(\frac{E_{i}}{E_{p}}, E_{p}\right) \frac{d E_{p}}{E_{p}},
$$

where $i$ could be $\gamma$ or $\nu$, and $F_{i}$ is the spectrum of the secondary $\gamma$ or $\nu$ in a single collision. This presentation works for $E_{p} \gtrsim 100 \mathrm{GeV}$, while for $E_{p}<100 \mathrm{GeV}$ a $\delta$-functional approximation for the energy of produced pions can be used to obtain the secondary spectrum (Kelner et al. 2006), i.e.,

$$
\begin{aligned}
\mathcal{F}_{i}\left(E_{i}\right)= & 2 c n_{\mathrm{sg}} \frac{\tilde{n}}{K_{\pi}} \int_{E_{i, \text { min }}}^{\infty} \sigma_{p p}\left(m_{p}+\frac{E_{\pi}}{K_{\pi}}\right) \\
& \times \frac{d N_{p}}{d E_{p}}\left(m_{p}+\frac{E_{\pi}}{K_{\pi}}\right) \frac{d E_{\pi}}{\sqrt{E_{\pi}^{2}-m_{\pi}^{2}}},
\end{aligned}
$$

where $E_{\pi}$ is the energy of pions and the pion rest mass $m_{\pi} \simeq 135 \mathrm{MeV}$ for gamma-ray production and $m_{\pi} \simeq 140 \mathrm{MeV}$ for neutrino production. $E_{i \text {,min }}=E_{i} / \zeta_{i}+\zeta_{i} m_{\pi}^{2} / 4 E_{i}$, with $\zeta_{\gamma}=$ 1 and $\zeta_{\nu}=1-m_{\mu}^{2} / m_{\pi}^{2}=0.427\left(m_{\mu} \simeq 106 \mathrm{MeV}\right.$ is the muon rest mass), $K_{\pi}=0.17$, and $\tilde{n}$ is a free parameter that is determined by the continuity of the flux of the secondary particle at $100 \mathrm{GeV}$. To get the differential isotropic gamma-ray luminosity for the shock front at $R$, we need to integrate over 

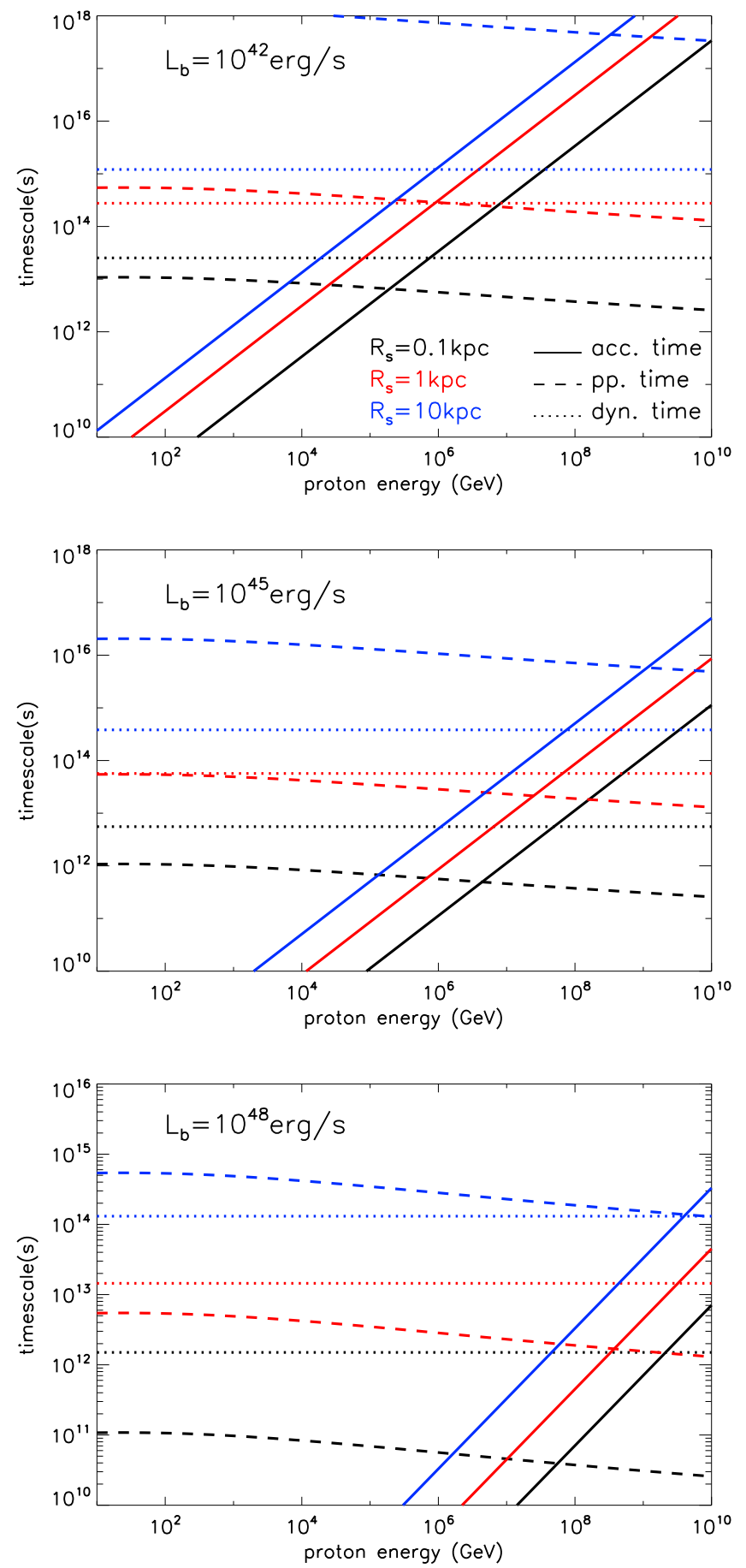

Figure 4. Timescales of different processes for AGNs with bolometric luminosities $L_{b}=10^{42} \mathrm{erg} \mathrm{s}^{-1}$ (top panel), $L_{b}=10^{45} \mathrm{erg} \mathrm{s}^{-1}$ (middle panel), and $L_{b}=10^{48} \mathrm{erg} \mathrm{s}^{-1}$ (bottom panel). Solid, dashed, and dotted lines represent acceleration, $p p$ reaction, and dynamical timescales, respectively. Black, red, and blue lines are for different shock radii, as marked inside the top panel.

the emission of all the protons injected in history, i.e.,

$$
L_{\gamma}\left(E_{\gamma}, R\right)=E_{\gamma}^{2} \int_{0}^{R} \mathcal{F}_{\gamma}\left\{N_{p}\left(E_{p}, r ; R\right), n_{\mathrm{sg}}(R)\right\} 4 \pi r^{2} d r,
$$

where $N_{p}\left(E_{p}, r ; R\right)$ represents the differential number density of protons that were injected at a radius $r(<R)$ when the shock front is at $R$. Note that $N_{p}\left(E_{p}, r ; R\right)$ is different from $N_{p}^{\text {inj }}\left(E_{p}, R\right)$ because of energy losses due to $p p$ interactions and adiabatic

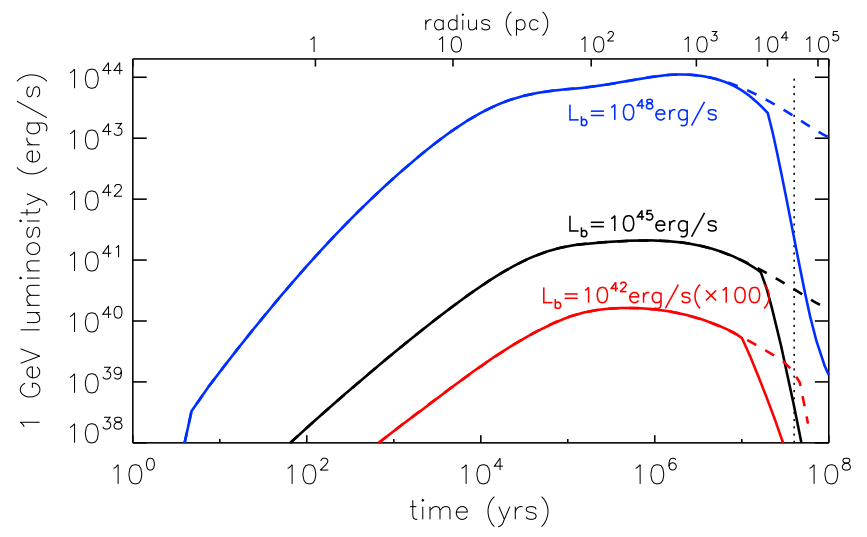

Figure 5. Light curve of $1 \mathrm{GeV}$ photons produced in an AGN-driven wind, with the AGN's bolometric luminosity being $10^{45} \mathrm{erg} \mathrm{s}^{-1}$ (black), $10^{42} \mathrm{erg} \mathrm{s}^{-1}$ (red), and $10^{48} \mathrm{erg} \mathrm{s}^{-1}$ (blue), located at $z=1$. The solid curves show the light curves corresponding to the results with the gas density profile and evolution presented in Figure 2, while dashed curves correspond to those in Figure 3. The vertical dotted line shows the Salpeter time $t_{\text {sal }}=4 \times 10^{7}$ yr. The top $x$-axis marks the corresponding shock radius at a certain time for $L_{b}=10^{45} \mathrm{erg} \mathrm{s}^{-1}$.

losses that will turn out to be important. After some approximations (see Appendix B for details), we arrive at

$$
\begin{aligned}
N_{p}\left(E_{p}, r ; R\right)= & N_{p}^{\mathrm{inj}}\left(E_{p}, r\right) \exp \left[-\left(1-2^{1-\Gamma_{\mathrm{CR}}}\right) \tau_{p p}\left(E_{p}, r, R\right)\right. \\
& -\left(\Gamma_{\mathrm{CR}}-1\right) \tau_{\mathrm{ad}}(r, R),
\end{aligned}
$$

where $\quad \tau_{p p}\left(E_{p}, r, R\right)=\int_{t}(r)^{t}(R) \sigma_{p p}\left(E_{p}\right) n_{\mathrm{sg}}\left(r^{\prime}(t)\right) c d t \quad$ and $\tau_{\text {ad }}(r, R)=\int_{t}(r)^{t}(R) v_{s}(t) d t / r^{\prime}(t)$. We note that photopion production interactions between accelerated protons and radiation fields of AGNs could be important at small radii, especially in the presence of AGN jets. However, we neglect this process because the number of accelerated protons at small radii is quite limited and also because this phase will not last long since the shock speed is high.

Then, the gamma-ray light curve of an AGN wind can be calculated based on the above equations, and the results are shown in Figure 5. The solid curves represent the $1 \mathrm{GeV}$ gamma-ray light curve produced by an AGN wind under the evolution and gas density profile presented in Figure 2 . The top $x$-axis marks the corresponding shock radius at a certain time for $L_{b}=10^{45} \mathrm{erg} \mathrm{s}^{-1}$. The gamma-ray luminosities reach the maximum when the shocks propagate to the radius of $\sim 0.1-1 \mathrm{kpc}$. The light curve also shows a plateau-like behavior in this range because the swept-up shell is a proton calorimeter, while the cosmic-ray luminosity is more or less constant in this range. The wind is not well decelerated at smaller radii, while the gas density becomes very small at larger radii. As a result, the neutrino and gamma-ray luminosities are relatively low at these locations (see Figure 4 for reference). We find that most energies $(\sim L t)$ are emitted when the shock is around $\lesssim 10 \mathrm{kpc}$. The average luminosity within $t_{\mathrm{sal}}$ is about 5 times smaller than the peak luminosity for $L_{b}=10^{42} \mathrm{erg} \mathrm{s}^{-1}, 3$ times smaller for $L_{b}=10^{45}$ and $10^{48} \mathrm{erg} \mathrm{s}^{-1}$. For reference, the dashed curves show the results in the case in which no break appears in the gas density profile in the halo, i.e., corresponding to the case presented in Figure 3. The average luminosity in this unrealistic case increases only by a factor of $\sim 2$. We note that the neutrino light curve should follow the same temporal behavior as that of the gamma ray.

Given the setup in this work, the AGN redshift (z) only influences the virial radius $R_{\text {vir }}$ and the correlated disk radius 
$R_{\text {disk }}=0.04 R_{\text {vir }}$. For the same AGN luminosity, a larger redshift leads to a smaller $R_{\text {vir }}$ and $R_{\text {disk }}$. As a result, the total mass content is reduced in the halo while the gas distribution is still the same in the disk. Therefore, a larger redshift leads to a less efficient gamma-ray/neutrino production in the halo. From the perspective of the light curve, the position of the decline in the light curve at $\lesssim 10 \mathrm{kpc}$ should appear earlier for larger $z$ and vice versa. In reality, the density may also positively scale as redshift and results in a larger gamma-ray/neutrino production for higher-redshift AGN host galaxies. In principle, a more careful treatment is necessary, such as done in Yuan et al. (2018).

We are aware that after an AGN shuts off, the forward shock may still expand into the ambient gas and accelerate protons. However, the host galaxy would no longer be regarded as a quasar-type or Seyfert-type AGN for the current observers, although it may be left as a low-luminosity AGN with powerful jets. Since we are only concerned with the gamma-ray and neutrino fluxes from AGNs, we do not consider the production beyond $t_{\mathrm{sal}}$. On the other hand, even if we assume that all the inactive galaxies were AGNs, their contribution to the diffuse gamma-ray and neutrino fluxes should be minor compared to that from AGNs at the present time. This is because the AGN fraction is about $\sim 1 \%$ among all the galaxies (Haggard et al. 2010), while the emissivity of gamma rays or neutrinos from an inactive galaxy is far smaller than $1 \%$ of the average emissivity during its active period.

\section{Contribution to Diffuse Neutrino and Gamma-ray Backgrounds}

In the previous section, we have examined the gamma-ray and neutrino light curves from a single AGN embedded in a dense ISM surrounded by a less dense halo. To obtain the diffusive gamma-ray/neutrino flux from AGNs throughout the universe, we need to sum up the contribution from AGNs with different luminosities and redshifts. Note that those AGN-driven wind bubbles should be at different stages of the evolution, so we need to take the average luminosity during their lifetime, which can be given by $\bar{L}_{\gamma / \nu}(E)=$ $\int_{0}^{t_{\text {Sal }}} L_{\gamma / \nu}[E, R(t)] d t / t_{\text {Sal }}$. Finally, we have the diffuse gammaray flux

$$
\begin{aligned}
E_{\gamma}^{2} \Phi_{\gamma}\left(E_{\gamma}\right)= & \frac{c}{4 \pi H_{0}} \iint \Psi\left(L_{b}, z\right) \frac{\bar{L}_{\gamma, L_{b}, z}\left[(1+z) E_{\gamma}, L_{b}\right]}{(1+z)^{2} E(z)} \\
& \times \exp \left[-\tau_{\gamma \gamma}\left(E_{\gamma}, z\right)\right] d z d L
\end{aligned}
$$

where $E(z)=\sqrt{(1+z)^{3} \Omega_{M}+\Omega_{\Lambda}}$ and $\Psi\left(L_{b}, z\right)$ is given by Hopkins et al. (2007),

$$
\Psi\left(L_{b}, z\right) \equiv \frac{d \Psi}{d \log L_{b}}=\frac{\Psi_{\star}}{\left(L_{b} / L_{\star}\right)^{\gamma_{1}}+\left(L_{b} / L_{\star}\right)^{\gamma_{2}}},
$$

accounting for the number of AGNs per logarithmic luminosity interval per volume. We adopt the pure luminosity evolution model, and the parameters are given by $\log \left(\Phi_{\star} / \mathrm{Mpc}^{3}\right)=-4.733$, $\log L_{\star}=\log L_{0}+k_{L, 1} \xi+k_{L, 2} \xi^{2}+k_{L, 3} \xi^{3}, \quad \xi=\log [(1+z) /$ $\left.\left(1+z_{\text {ref }}\right)\right], z_{\text {ref }}=2, \log \left(L_{0} / L_{\odot}\right)=12.965, k_{L, 1}=0.749, k_{L, 2}=$ $-8.03, k_{L, 3}=4.40, \gamma_{1}=0.517$, and $\gamma_{2}=0.296 . \tau_{\gamma}\left(E_{\gamma}, z\right)$ is the gamma-ray opacity due to absorption by cosmic microwave

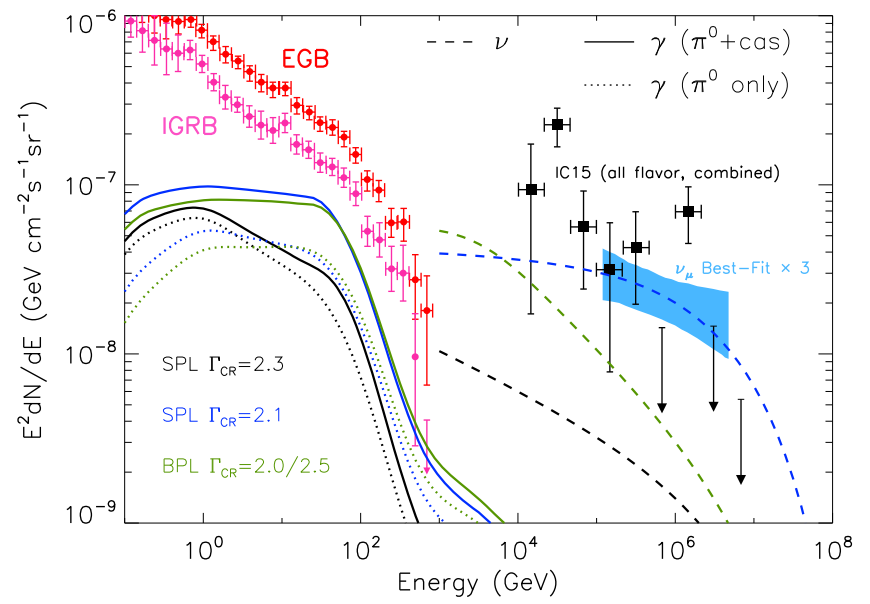

Figure 6. Diffuse gamma-ray flux (solid curves) and all-flavor neutrino flux (dashed curves). The gamma-ray flux from direct $\pi^{0}$ decay is also shown (dotted curves). Different colors represent the cases for different proton spectral indices. The EBL model by Finke et al. (2010) is adopted. The red and pink filled circles represent the Fermi-LAT EGB and IGRB data for foreground model A, respectively (Ackermann et al. 2015). The black filled squares are the astrophysical neutrino fluxes measured by IceCube (Aartsen et al. 2015), obtained from a combined maximum likelihood analysis, while the blue shaded region corresponds to the $68 \%$ C.L. allowed region for the muon (including anti-muon) neutrino flux with an SPL model (IceCube Collaboration et al. 2017; the original data have been multiplied by 3 to convert to an allflavor flux, assuming a flavor ratio of $1: 1: 1$ ).

background $(\mathrm{CMB})$ and extragalactic background light (EBL) for a photon that originated from redshift $z$ with a redshifted energy $E_{\gamma}$ at Earth. We adopt an EBL model of moderate intensity provided by Finke et al. (2010). In WLI and WLII, they adopted the EBL model of Stecker et al. (2006), which was already ruled out by the gammaray observations by Fermi-LAT and observations with imaging atmospheric Cherenkov telescopes (e.g., Abdo et al. 2010; Orr et al. 2011). But to compare with their results, we also adopt this EBL model in our calculation for reference. Note that one should remove this term when calculating the diffuse neutrino flux.

After integrating over the luminosity in the range of $10^{42}-10^{48} \mathrm{erg} \mathrm{s}^{-1}$ and redshift in the range of $z=0-5$, we can obtain the diffusive gamma-ray and neutrino backgrounds. Figure 6 shows the results with different proton spectra at injection, i.e., SPL spectrum with $\Gamma_{\mathrm{CR}}=2.3$ and $\Gamma_{\mathrm{CR}}=2.1$, and BPL spectrum with $\Gamma_{\mathrm{CR}}=2$ below $100 \mathrm{TeV}$ and $\Gamma_{\mathrm{CR}}=2.5$ above $100 \mathrm{TeV}$. No internal absorption of high-energy photons is considered, but electromagnetic cascades initiated by highenergy photons during the propagation in the intergalactic space are taken into account based on the EBL model of Finke et al. (2010). In this work, the calculation of electromagnetic cascades follows the simplified method described in Liu et al. (2016), and a sufficiently weak intergalactic magnetic field $(\lesssim 1 \mathrm{nG})$ is assumed so that cascades in the considered energy range will not be affected by synchrotron losses (see Murase et al. 2012). Given the total cosmic-ray luminosity, the $\mathrm{GeV}$ gamma-ray flux from direct $\pi^{0}$ decay in the case of $\Gamma_{\mathrm{CR}}=2.3$ is higher than those in the cases of $\Gamma_{\mathrm{CR}}=2.1$ and the BPL case. However, due to the contribution of the cascade emission whose energy production rate is $\gtrsim 100 \mathrm{GeV}$ gamma-ray photons, the total $\mathrm{GeV}$ gamma-ray flux for $\Gamma_{\mathrm{CR}}=2.3$ becomes smaller than the latter two cases. 
Neutrinos are not affected during their propagation, except for adiabatic losses due to the expansion of the universe. Thus, if one extrapolates neutrino flux to the $\mathrm{GeV}$ range, the flux level is consistent with the $\pi^{0}$ gamma-ray flux. We can see that, in all three cases, the gamma-ray fluxes are significantly lower than the observed EGB flux at $1-10 \mathrm{GeV}$, constituting at most a fraction of $\lesssim 30 \%$ of the EGB around $50 \mathrm{GeV}$. On the other hand, the neutrino fluxes are lower than the best-fit IceCube flux at $10 \mathrm{TeV}$ by a factor of 5-20. However, we note that in the case of a hard index, $\Gamma_{\mathrm{CR}}=2.1$, although the neutrino flux is about 5 times lower than the best-fit value at $10 \mathrm{TeV}$, the flux above $100 \mathrm{TeV}$ is consistent with that inferred from throughgoing muon neutrino detection (assuming the neutrino flavor ratio to be 1:1:1). Indeed, the two-component scenario is possible, in which a hard component above $100 \mathrm{TeV}$ (Aartsen et al. 2015, 2016) can be explained by cosmic-ray reservoir models, which may be even related to the sources of ultra-highenergy cosmic rays (Liu et al. 2014; Murase \& Waxman 2016; Fang et al. 2017).

Given the adopted luminosity function, we find that AGNs with luminosity around $10^{45} \mathrm{erg} \mathrm{s}^{-1}$ make the most important contribution. This explains the softening of the neutrino spectrum at $\sim 1 \mathrm{PeV}$ since the maximum proton energy is around $100 \mathrm{PeV}$ for $L=10^{45} \mathrm{erg} \mathrm{s}^{-1}$ AGNs when the shock is around $10 \mathrm{kpc}$ (see Figure 4), where most energies are released as we discussed above. We reiterate that acceleration of $\sim 1-100 \mathrm{PeV}$ protons is required to produce $10 \mathrm{TeV}-1 \mathrm{PeV}$ neutrinos via inelastic $p p$ collisions. According to Figure 4, protons with such high energies may not be achieved in the forward shocks of some AGN winds, especially when considering that the Bohm diffusion fails to establish. A more realistic diffusion model would result in a much smaller maximum proton energy, probably leading to a cutoff in the produced neutrino spectrum below $10 \mathrm{TeV}$.

\subsection{Comparison to Previous Works}

To compare our results with those in the previous literature, we consider a case with the proton spectral index of $\Gamma_{\mathrm{CR}}=2.3$, counting only the gamma-ray flux from $\pi^{0}$ decay, and employ the EBL model given by Stecker et al. (2006), which are adopted in WLI and WLII. The result is shown in Figure 7. Our gamma-ray flux is several times lower than that obtained in WLI. This would be partly because they extrapolated an $R^{-2}$ profile for the gas density down to the smallest radius. Such a profile leads to the injection of a huge amount of protons and a high $p p$ collision efficiency. Also, it seems that their $1 \mathrm{GeV}$ gamma-ray luminosity exceeds that of the kinetic luminosity of the wind at early stages. Our work takes into account the proton cooling due to inelastic collisions and adiabatic losses. Whereas the light curve in WLII decreases with time at early stages (see Figure 2 in WLII), we expect that it is rather flat when the system is calorimetric in high-density regions and the injection of cosmic rays is supposed to be constant (see the previous section and discussions in Lamastra et al. 2017). There is also a difference in the gamma-ray spectral shape between our result and that in WLI and WLII. In our calculation, the cutoff in the gamma-ray flux appears at a higher energy than that shown in WLI and WLII. The EBL cutoff should be around $10-20 \mathrm{GeV}$, so the cutoff shown in WLI and WLII should not be caused by the EBL absorption. We do not show the neutrino flux since their the neutrino flux shown in WLI

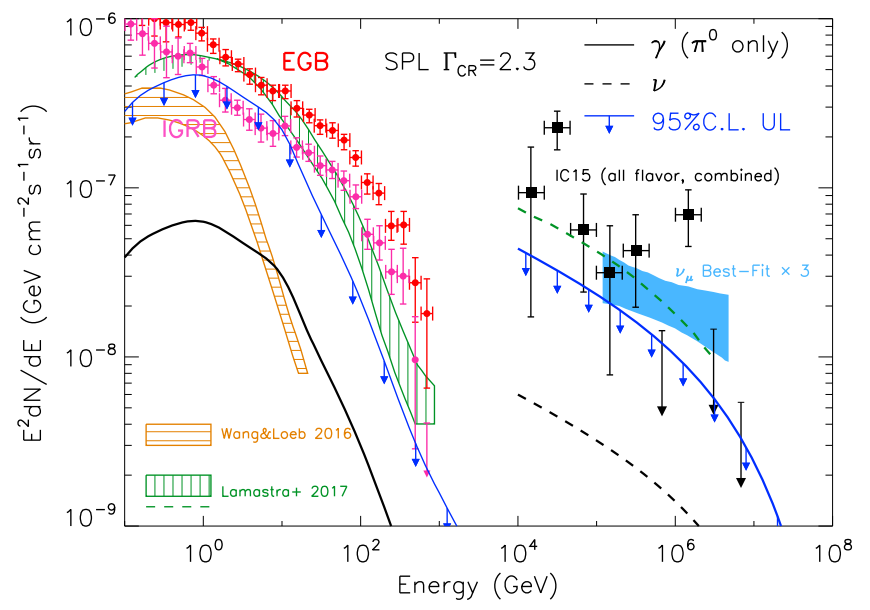

Figure 7. Comparison of our result to the results in the previous literature. An SPL with an index of $\Gamma_{\mathrm{CR}}=2.3$ and the EBL model provided by Stecker et al. (2006) are employed. The gamma-ray contribution from electromagnetic cascades is not considered. The blue curves with downward-pointing arrows present the upper limits for gamma-ray and neutrino fluxes when the 95\% C.L. of the IGRB data is not violated. The yellow hatched region (dashed curve) is the gamma-ray (neutrino) flux obtained by WLI, while the green hatched region (dashed curve) is the gamma-ray (neutrino) flux obtained by Lamastra et al. (2017).

largely deviates from the theoretical expectation for the relationship between neutrino and gamma-ray fluxes. The gamma-ray and neutrino fluxes generated in $p p$ collisions should be roughly comparable at $E_{\gamma} \approx 2 E_{\nu}$. The gamma-ray flux at $1 \mathrm{GeV}$ and all-flavor neutrino flux at $10 \mathrm{TeV}$ have a difference of $\approx(3 / 2)(20 \mathrm{TeV} / 1 \mathrm{GeV})^{2-\Gamma} \simeq 0.08$ for an SPL proton spectrum with $\Gamma_{\mathrm{CR}}=2.3$. This agrees with our result and the result of Lamastra et al. (2017), while the result in WLI indicates that the neutrino flux at $10 \mathrm{TeV}$ is comparable to the gamma-ray flux at $1 \mathrm{GeV}$.

The results of Lamastra et al. (2017) are consistent with ours in terms of the spectral shapes of gamma-ray and neutrino emissions. However, their fluxes are about one order of magnitude larger than ours. Similarly to WLI and WLII, Lamastra et al. (2017) also extrapolated an $R^{-2}$ profile for the gas density to very small radii, making the galactic disk a proton calorimeter. But since they considered the cooling of the accelerated protons due to $p p$ collisions, we do not see a large difference due to this extrapolation. On the other hand, all the accelerated protons are well confined and expand with the shock in our calculation, and the adiabatic cooling reduces the fluxes by a factor of $\sim 2$. In contrast, Lamastra et al. (2017) assumed that all the protons escape the shock and hence do not suffer from adiabatic losses. However, in reality, the escaping protons interact with the uncompressed gas with a smaller density, and they may also diffuse to a larger radius, where the gas density is very low. These effects are not considered in their calculation. If the diffusion coefficient is large, the $p p$ optical depth can be lowered by a factor of a few. On the other hand, if the diffusion coefficient is too small, the escaped protons could be caught up by the shock, implying that they cannot escape in the first place. Another important cause for the difference is that the shock expansion in the galaxy occurs for a short time. According to our calculation, the time that the forward shock experiences in the galactic disk is much shorter than the time in the halo. On the other hand, the total lifetime of AGNs (i.e., the Salpeter time) is about $\sim 10^{7} \mathrm{yr}$, which is much shorter than the age of the galaxy. Thus, for the current 
observer, it is unlikely that the forward shocks in all the host galaxies throughout the universe are currently located in their galactic disks. To evaluate the contribution from all AGNdriven winds in the universe, it is more appropriate to first average the gamma-ray/neutrino luminosity over the entire evolution time and then sum up over redshifts, as done by WLI, WLII, and this work. Note that the gamma-ray and neutrino luminosities from the shock in the halo should be lower owing to the much lower gas density, so this can further lower the diffusive flux by another factor of 3-5. Lastly, Lamastra et al. (2017) adopted a different luminosity function and redshift evolution of AGNs.

The employed luminosity function includes both radio-quiet AGNs and radio-loud AGNs. Radio-loud AGNs are accompanied by powerful jets, which may have contributions to the diffuse neutrino background (Murase et al. 2008, 2013; Becker Tjus et al. 2014; Hooper 2016) by the interactions inside largescale structures, or by interactions in the AGN core regions. Note that AGN winds and jets are not mutually exclusive. Our result does not exclude the possibility of these powerful jets as the sources of high-energy neutrinos.

\section{Implications for the Diffuse Neutrino Background}

Obviously, the AGN-wind model has large model uncertainties, which are difficult to estimate. In principle, one could increase the neutrino flux to fit IceCube's observation with extreme parameters, by, for example, assuming a larger ratio of the wind's kinetic luminosity to AGN's bolometric luminosity and a larger fraction of the thermal energy converted to nonthermal energy of the accelerated protons in the shock. However, the gamma-ray flux will then be increased by the same level, approximately matching the flux of EGB but overshooting the flux of IGRB (Ackermann et al. 2015). Indeed, a strong constraint on the sources of the cumulative neutrino background is unavoidable, as found by Murase et al. (2013). There are four facts: (1) the measured energy flux of neutrinos at $10-30 \mathrm{TeV}$ is comparable to that of IGRB at $100 \mathrm{GeV}$, say, $10^{-7} \mathrm{GeV} \mathrm{cm}^{-2} \mathrm{~s}^{-1} \mathrm{sr}^{-1}$; (2) the gamma-ray flux and neutrino flux simultaneously produced via $p p$ collisions are comparable beyond $1 \mathrm{GeV}$; (3) photons with energy above $100 \mathrm{GeV}$ will initiate electromagnetic cascades and form a diffuse gamma-ray background with an approximate $E^{-2}$ spectrum up to $\lesssim 100 \mathrm{GeV}$ and an energy flux similar to the injected one, provided that they are injected at cosmological distances (Strong \& Wolfendale 1973; Berezinskii \& Smirnov 1975; Berezinsky et al. 2011; Kachelrieß et al. 2012; Murase et al. 2012); (4) blazars, including the unresolved ones, contribute $86_{-14}^{+16} \%$ of the EGB above $50 \mathrm{GeV}$ (Ackermann et al. 2016; see also Lisanti et al. 2016) and $50_{-11}^{+12} \%$ below $10 \mathrm{GeV}$ (Ajello et al. 2015), shrinking the room for the contribution of other sources to IGRB around $10 \mathrm{GeV}$ down to a level of at most $50 \%$. As a result, as shown by previous works, a strong tension against the IGRB data is unavoidable when such $p p$ scenarios explain the measured neutrino flux below $100 \mathrm{TeV}$ (e.g., Chang et al. 2016; Murase et al. 2016; Xiao et al. 2016), especially in the presence of cosmogenic gamma rays (Berezinsky \& Kalashev 2016; Liu et al. 2016; Murase \& Waxman 2016). Note that the medium-energy component is unlikely to be Galactic. The latest shower and medium-energy starting event analyses suggest that the arrival distribution of neutrinos with these energies is consistent with an isotropic distribution (IceCube Collaboration et al.
2017), and there is no evidence for a special source around the Galactic center, Fermi bubbles, and other structures such as Loop I. In addition, HAWC has already given a strong limit on the diffuse gamma-ray flux, $E_{\gamma}^{2} \Phi_{\gamma} \sim(0.3-1) \times$ $10^{-7} \mathrm{GeV} \mathrm{cm}^{-2} \mathrm{~s}^{-1} \mathrm{sr}^{-1}$, in the $30-100 \mathrm{TeV}$ range around the Galactic halo region (Abeysekara et al. 2017, 2018). Such diffuse gamma-ray limits can constrain $p p$ scenarios, in which a significant fraction of IceCube neutrinos are explained by Galactic sources (Ahlers \& Murase 2014). For example, if the Fermi bubbles (Fang et al. 2017; Sherf et al. 2017) or Loop I (Andersen et al. 2017) dominantly contribute to the 10-100 TeV neutrino flux, the diffuse gamma-ray flux from the sky region $\Delta \Omega$ is expected to be $E_{\gamma}^{2} \Phi_{\gamma} \sim 4 \times$ $10^{-7}(3 \mathrm{sr} / \Delta \Omega) \mathrm{GeV} \mathrm{cm} \mathrm{cm}^{-2} \mathrm{~s}^{-1} \mathrm{sr}^{-1}$, which is higher than the existing limits. Thus, the Galactic origin of these diffuse isotropic 10-100 TeV neutrinos is unlikely.

Thus, we focus on the extragalactic interpretation of IceCube neutrinos. Taking the case of $\Gamma_{\mathrm{CR}}=2.3$ as an example, we perform the chi-square analysis and place a limit on AGNdriven wind models. For our model, we find that in order not to violate the $95 \%$ C.L. of the IGRB data at each energy bin, ${ }^{9}$ the gamma-ray flux can be shifted upward by a factor of 7.3 at most if the amplitude of the flux is taken to be a free parameter and the spectral shape is fixed. The neutrino flux will be increased by the same factor, resulting in a flux of $4.2 \times 10^{-8} \mathrm{GeV} \mathrm{cm}^{-2} \mathrm{~s}^{-1} \mathrm{sr}^{-1}$ at $10 \mathrm{TeV}$, which is still about 5 times smaller than the best-fit flux of the IceCube neutrinos at $10 \mathrm{TeV}$. This upper limit for our model template is shown with the blue curves with downward-pointing arrows in Figure 7. Considering the uncertainty in the measurement of IGRB due to the Galactic gamma-ray foreground, IGRB flux can only be increased by a factor of $\sim 1.5$, which only slightly changes the result here. The spectral shape of diffuse gamma-ray flux obtained by Lamastra et al. (2017) is very similar to ours, so the most constraining energy bin of the IGRB (the one in $4.5-6.4 \mathrm{GeV}$ ) should be the same as the one in our model. Therefore, the obtained upper limit for our model should also apply to the model of Lamastra et al. (2017). This is consistent with the multimessenger constraints obtained by Murase et al. (2013) for $p p$ scenarios, which showed that the spectral index cannot be softer than $\Gamma \sim 2.1-2.2$ in order not to overshoot the IGRB data, when the neutrino flux is normalized to $100 \mathrm{TeV}$. Note that the constraint becomes stricter if one normalizes the neutrino flux to the observation at $10 \mathrm{TeV}$, ruling out even a harder slope. Indeed, for $\Gamma_{\mathrm{CR}}=2.1$, we find that the gammaray flux can be increased only by a factor of 2 at most in order not to violate the IGRB data, and the neutrino flux in this limit is still only $\sim 30 \%-40 \%$ of the best-fit flux at $10 \mathrm{TeV}$. Although harder spectra would help to alleviate the tension, the observed neutrino spectrum in the $10-100 \mathrm{TeV}$ range is too soft to explain with such hard spectra. This tension becomes severer if we recall that a large fraction of the IGRB is attributed to blazars. Thus, the AGN-driven winds cannot be the dominant sources for $\gtrsim 10 \mathrm{TeV}$ neutrinos, unless the sources are opaque to $10-100 \mathrm{GeV}$ photons (which implies hidden cosmic-ray accelerators; Murase et al. 2016). However, since most gamma rays are emitted at $\lesssim 10 \mathrm{kpc}$ according to Figure 5 , it is unlikely

\footnotetext{
9 The one-sided 95\% C.L. upper limit of the IGRB at the $i$ th energy bin is calculated by $F_{95 \% \text { U.L. }}\left(E_{i}\right)=F_{\mathrm{IGRB}}\left(E_{i}\right)+\sqrt{\chi_{90 \%}^{2}} \sigma_{\mathrm{IGRB}}\left(E_{i}\right)$, where $F_{\mathrm{IGRB}}\left(E_{i}\right)$ is the measured IGRB flux at the $i$ th energy bin and $\sigma_{\mathrm{IGRB}}\left(E_{i}\right)$ is the statistical error of the IGRB flux at the $i$ th energy bin. $\chi_{90 \%}^{2}=2.71$ is the chi-square value for $90 \%$ C.L. for one degree of freedom.
} 


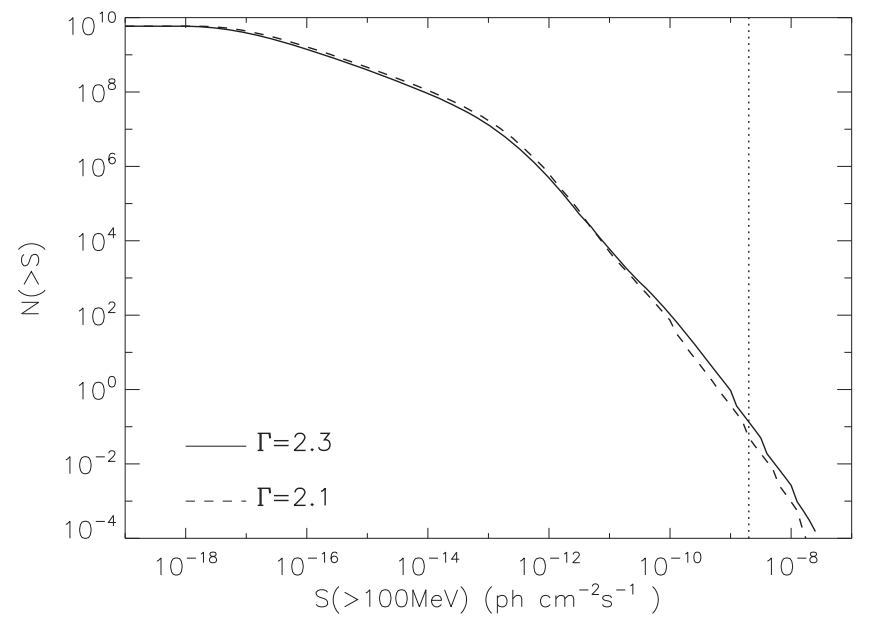

Figure 8. Cumulative source count distribution of the gamma-ray flux above $100 \mathrm{MeV}$ from AGN-driven winds. The vertical dotted line is the $5 \sigma$ integrated sensitivity for a point source with a photon index of $\Gamma=2.0$.

that an intense soft X-ray photon field would appear at this radius and effectively absorb $10-100 \mathrm{GeV}$ gamma-ray photons inside the host galaxy.

In addition, we note that only gamma rays from $\pi^{0}$ decay are considered, as in the analysis of Murase et al. (2013). Given a hard proton spectrum $\Gamma_{\mathrm{CR}} \lesssim 2.1$, we can expect the $\mathrm{GeV}$ gamma-ray flux that cascaded from higher energies to be comparable to or even more important than that from $\pi^{0}$ decay at $\mathrm{GeV}$ energies if the internal absorption of gamma rays is not very important (which is valid in the AGN-wind case). This can be seen from the $\Gamma_{\mathrm{CR}}=2.1$ case (blue curves) in Figure 6 . Thus, even if only neutrinos above $100 \mathrm{TeV}$ are ascribed to certain species of extragalactic sources with a hard injection proton spectrum, the accompanying $\mathrm{GeV}$ gamma-ray flux would reach a level of $10^{-7} \mathrm{GeV} \mathrm{cm}^{-2} \mathrm{~s}^{-1} \mathrm{sr}^{-1}$ if there is little internal absorption of these gamma rays. The current analysis of the blazar contribution to the IGRB still has some uncertainties. If future measurements can lower the nonblazar component of the IGRB to half of the current level or even below, some of our model assumptions, including various other models for $\gtrsim 100 \mathrm{TeV}$ neutrinos, could be tested critically.

\section{Implications for Point-source Detection}

AGN-driven winds are predicted to be persistent gamma-ray emitters, and current gamma-ray detectors such as Fermi-LAT should be able to detect them with a long-term exposure. The $5 \sigma$ integrated sensitivity for 108 months of LAT observations on a high-latitude point source above $100 \mathrm{MeV}$ with a photon index of -2.0 is ${ }^{10} S_{\lim }(>100 \mathrm{MeV}) \approx 10^{-9}$ photons $\mathrm{cm}^{-2} \mathrm{~s}^{-1}$. We calculate the cumulative source count distribution $N(>S)$ based on the cases of $\Gamma_{\mathrm{CR}}=2.1$ and $\Gamma_{\mathrm{CR}}=2.3$ shown in Figure 6, with $S$ here being the photon flux in units of photons $\mathrm{cm}^{-2} \mathrm{~s}^{-1}$ above $100 \mathrm{MeV}$ from a certain source. The result is shown in Figure 8. As can be seen, the number of detectable sources is about unity. A longer-time (e.g., $10 \mathrm{yr}$ ) monitoring would help to discover point sources. If the AGNdriven winds give the dominant contribution to the IGRB, a few sources can be detected by Fermi-LAT, which is consistent

\footnotetext{
${ }^{10}$ We obtain this sensitivity by multiplying a factor of $\sqrt{2 / 9}$ by the $2 \mathrm{yr}$ sensitivity, which is given in https://fermi.gsfc.nasa.gov/ssc/data/analysis/ documentation//Cicerone/Cicerone_LAT_IRFs/LAT_sensitivity.html.
}

with the previous constraint on the effective source number density (Murase \& Waxman 2016). Our result is also consistent with the fact that a starburst-coexisting AGN, NGC 1068, was detected by Fermi-LAT.

Detecting individual neutrino sources with IceCube seems difficult. However, IceCube-Gen2 will be able to detect highenergy neutrino signals from most of the known astrophysical sources, including galaxies with AGN-driven winds. Murase \& Waxman (2016) investigated detection prospects for nearby high-density galaxies and argued that NGC 1068 is one of the promising target sources for IceCube-Gen2.

\section{Summary}

In this work, we studied the diffuse gamma-ray and neutrino fluxes produced by AGN-driven winds. The expansion of the AGN-driven winds into the ISM gas and halo gas in the AGN's host galaxy will form a wind bubble, which can accelerate protons at the forward shock. Gamma rays and neutrinos are produced through the decay of pions, which can be generated via inelastic $p p$ collisions between the accelerated protons and the shocked gas. We solve the dynamic evolution of the wind from the disk to the halo of the host galaxy. Based on the evolution model, taking into account some details such as proton cooling processes, we calculated the proton cooling and resulting pion production processes. The diffuse gamma-ray and neutrino fluxes are obtained by summing up all possible contributions from AGNs in the universe. We found that the generated gamma-ray flux can account for $\lesssim 30 \%$ of the EGB flux around $50 \mathrm{GeV}$. For $\Gamma_{\mathrm{CR}}=2.3$, the resulting neutrino flux is several times lower than or even more than one order of magnitude lower than the observed IceCube flux at $10 \mathrm{TeV}$. Given that model uncertainties are large, the neutrino flux may be increased several times with optimistic (but somewhat extreme) model parameters. However, independent of details of the models, the IGRB data already rule out the possibility that the dominant fraction of IceCube neutrinos is accounted for in this model, for soft indices of $\Gamma \gtrsim 2.2$. This conclusion can be strengthened if the contribution of unresolved blazars is taken into account. However, with hard spectral indices of $\Gamma \lesssim 2.2$, it is still possible to explain the higher-energy component of the diffuse neutrino flux above $\sim 0.1 \mathrm{PeV}$. Another potential constraint on the contribution of neutrinos from AGN winds is the synchrotron radiation of coproduced secondary electrons in the shocks, which are supposed to give rise to multiwavelength radiation at a similar flux level. The predicted multiwavelength fluxes can be compared to the observation, and relevant physical quantities may be constrained. In addition, primary electrons can also be accelerated in the shocks, and their radiation may provide additional information about the parameters (Jiang et al. 2010; Wang \& Loeb 2015).

In the AGN-driven wind model we consider, one should keep in mind that the starburst activity and AGN activity are not mutually exclusive. In cosmic-ray reservoir models, multiple classes of cosmic-ray accelerators are generally allowed, and supernovae, hypernovae, AGN-driven outflows, and possible weak jets from AGNs (although jets may be relevant for powerful radio-loud quasars) may contribute as accelerators of cosmic rays in the $1-100 \mathrm{PeV}$ range (Tamborra et al. 2014). The model also predicts that a few nearby sources can be detected by Fermi-LAT, which seems consistent with the detection of NGC 1068. 
We thank Dr. Xiawei Wang and Prof. Walter Winter for helpful discussion, as well a Prof. Jeremiah Ostriker for his insightful comment. This work is supported by the 973 program under grant 2014CB845800 and the NSFC under grants 11625312 and 11273016 . The work of K.M. is supported by the Alfred P. Sloan Foundation and NSF grant no. PHY-1620777. R.-Y.L. thanks Prof. Huirong Yan for her friendly hospitality during his visit in DESY.

\section{Appendix A \\ Dynamic Evolution of the AGN-wind Bubble}

\section{A.1. The Relation between AGN's Bolometric Luminosity and Dark Matter Halo Mass}

One can check the details in Wang \& Loeb (2015) and references therein; we just follow and summarize their treatment here for convenience. AGN's bolometric luminosity is assumed to be a fraction $f_{\mathrm{AGN}}=0.5$ of the Eddington luminosity of the SMBH $L_{\mathrm{Edd}}=1.38 \times 10^{38}\left(M_{\mathrm{BH}} / M_{\odot}\right) \mathrm{erg} \mathrm{s}^{-1}$. The mass of the SMBH is related to the bulge stellar mass by (McConnell \& Ma 2013)

$$
\log \left(M_{\mathrm{BH}} / M_{\odot}\right)=8.46+1.05 \log \left(\frac{M_{\text {bulge }}}{10^{11} M_{\odot}}\right) .
$$

On the other hand, they adopt a bulge-to-total stellar mass ratio $B / T=M_{\text {bulge }} / M_{\star}$ of 0.3 . Finally, one can obtain the dark matter halo mass through the relation (Moster et al. 2010)

$$
M_{\star}=M_{\star, 0} \frac{\left(M_{\text {halo }} / M_{1}\right)^{\gamma_{1}}}{\left[1+\left(M_{\text {halo }} / M_{1}\right)^{\beta}\right]^{\left(\gamma_{1}-\gamma_{2}\right) / \beta}},
$$

where $\log \left(M_{\star, 0} / M_{\odot}\right)=10.864, \log \left(M_{1} / M_{\odot}\right)=10.456, \quad \gamma_{1}=$ 7.17, $\gamma_{2}=0.201$, and $\beta=0.557$. Thus, given the AGN's bolometric luminosity, one can find the halo mass by the above equations. Note that an upper limit for $M_{\text {halo }}=10^{13} M_{\odot}$ is set, following WLII.

\section{A.2. Two-temperature Plasma Cooling in the Shocked Wind Region}

Following Faucher-Giguère \& Quataert (2012), we also consider the two-temperature effect in the plasma. In this scenario the shock heats protons and electrons to different temperatures (Equations (2) and (3)), with an initial difference of $m_{p} / m_{e}$. With a higher initial temperature, the Coulomb collision between protons and electrons will transfer thermal energy from protons to electrons until $T_{p}=T_{e}$. The timescale to reach such a equilibrium reads as (Faucher-Giguère \& Quataert 2012)

$$
t_{\mathrm{pe}}=\frac{3 m_{e} m_{p}}{8(2 \pi)^{1 / 2} n_{p, \mathrm{sw}} e^{4} \ln \Lambda}\left(\frac{k T_{e}}{m_{e}}+\frac{k T_{p}}{m_{p}}\right)^{3 / 2},
$$

with

$$
\ln \Lambda \simeq 39+\ln \left(\frac{T_{e}}{10^{10} \mathrm{~K}}\right)-\frac{1}{2} \ln \left(\frac{n_{e, \mathrm{sw}}}{1 \mathrm{~cm}^{-3}}\right),
$$

where $n_{p, \mathrm{sw}}=n_{e, \mathrm{sw}}=n_{\mathrm{sw}}$ is the proton number density in the shocked wind region given. In Faucher-Giguère \& Quataert (2012), the authors adopt an analytic approximate proton cooling timescale by assuming that Coulomb collision is the only process that changes the temperature of protons. However, as we mentioned in Section 2, the adiabatic expansion and the freshly injected protons from the reverse shock will also affect the proton temperature. Thus, instead of adopting the oversimplified approximation, we trace the temperature of protons and electrons separately during the evolution. Note that Equations (2) and (3) are only valid for the temperature immediately after the shock. The average temperature in the entire downstream region is more relevant to the evolution. Therefore, we only use Equations (2) and (3) as an initial temperature, while we calculate the proton temperature and electron temperature during the evolution by $T_{p}=P_{p, \mathrm{sw}} / n_{\mathrm{sw}} k$ and $T_{e}=P_{e, \mathrm{sw}} / n_{\mathrm{sw}} k$, respectively. The evolution of $P_{p, \mathrm{sw}}$ and $P_{e, \mathrm{sw}}$ can be found by Equations (10)-(12). In Equation (12), the Compton cooling/heating term $L_{c}$ is given by

$$
L_{c}=-\frac{d U_{\mathrm{ph}}}{d t} V_{\mathrm{sw}},
$$

where $V_{\mathrm{sw}}=4 \pi\left(R_{s}^{3}-R_{\mathrm{rs}}^{3}\right) / 3$ and the rate of energy transfer between the electrons and photons due to Compton scattering is given by (Sazonov \& Sunyaev 2001)

$$
\begin{aligned}
\frac{d U_{\mathrm{ph}}}{d t}= & 4 U_{\mathrm{AGN}} \sigma_{T} n_{e, \mathrm{sw}} c\left(\frac{k T_{e}}{m_{e} c^{2}}-\frac{k T_{X}}{m_{e} c^{2}}\right) \\
& \times\left(1+\frac{5}{2} \frac{k T_{e}}{m_{e} c^{2}}-2 \pi^{2} \frac{k T_{X}}{m_{e} c^{2}}\right) \\
& +4 U_{\mathrm{CMB}} \sigma_{T} n_{e, \mathrm{sw}} c\left(\frac{k T_{e}}{m_{e} c^{2}}-\frac{k T_{\mathrm{CMB}}}{m_{e} c^{2}}\right) \\
& \times\left(1+\frac{5}{2} \frac{k T_{e}}{m_{e} c^{2}}-2 \pi^{2} \frac{k T_{\mathrm{CMB}}}{m_{e} c^{2}}\right) .
\end{aligned}
$$

This formula is valid up to mildly relativistic electrons $\left(k T_{e} \leqslant 0.1 m_{e} c^{2}\right) . T_{X}$ is the Compton temperature of the radiation field of AGNs, which is found to be in a narrow range around $2 \times 10^{7} \mathrm{~K}$ (Sazonov et al. 2004), and $\sigma_{T}$ is the Thomson cross section. $U_{\mathrm{ph}}=U_{\mathrm{AGN}}+U_{\mathrm{CMB}}=2.8 \times 10^{-12}\left(L_{b} / 10^{45} \mathrm{erg} \mathrm{s}^{-1}\right)$ $(R / 10 \mathrm{kpc})^{-2}+4 \times 10^{-13}(1+z)^{4} \mathrm{erg} \mathrm{cm}^{-3}$. Besides the Compton scattering, electrons can also lose energy via free-free emission and synchrotron radiation as considered in Equation (12). The cooling time due to free-free emission for electrons is $t_{\mathrm{ff}}=\frac{3 n_{e} k T_{e}}{\epsilon_{\mathrm{ff}}}=4.7 \times 10^{8}\left(\frac{T_{e, \mathrm{w}}}{10^{10} \mathrm{~K}}\right)^{1 / 2} n_{e, \mathrm{sw}}^{-1} g_{B}^{-1} \mathrm{yr}$, where $\epsilon_{\mathrm{ff}}=1.4 \times 10^{-22} T_{e} / 10^{10} \mathrm{~K} \quad n_{e}^{2} g_{B} \mathrm{erg} \mathrm{cm}^{-3} \mathrm{~s}^{-1}$ is the thermal free-free emissivity, with $g_{B} \sim 1$ being the average Gaunt factor. The synchrotron cooling timescale is $t_{\mathrm{syn}}=1.6 \times$ $10^{12}\left(\frac{B}{1 \mu \mathrm{G}}\right)^{-2}\left(\frac{T_{e}}{10^{10} \mathrm{~K}}\right)^{-1}$ yr. We neglect the self-Compton scattering off the photons from free-free emission and synchrotron radiation here.

In Figure 9, we show the evolution of proton temperature and electron temperature in the case of $L_{b}=10^{42} \mathrm{erg} \mathrm{s}^{-1}, L_{b}=$ $10^{45} \mathrm{erg} \mathrm{s}^{-1}$, and $L_{b}=10^{48} \mathrm{erg} \mathrm{s}^{-1}$. Due to the continuous injection of freshly shocked wind, the temperature of protons and electrons cannot reach equilibrium before the AGN shuts off. The only exception is at very small radius when the wind has not been well decelerated, as can be seen around $R=0.1 \mathrm{pc}$ in the case of $L_{b}=10^{48} \mathrm{erg} \mathrm{s}^{-1}$ (for $L_{b}=10^{42}$ and $10^{45} \mathrm{erg} \mathrm{s}^{-1}$, this happens at a smaller radius than the initial radius considered in the calculation). The reverse shock is very weak, so that the temperature of shocked 

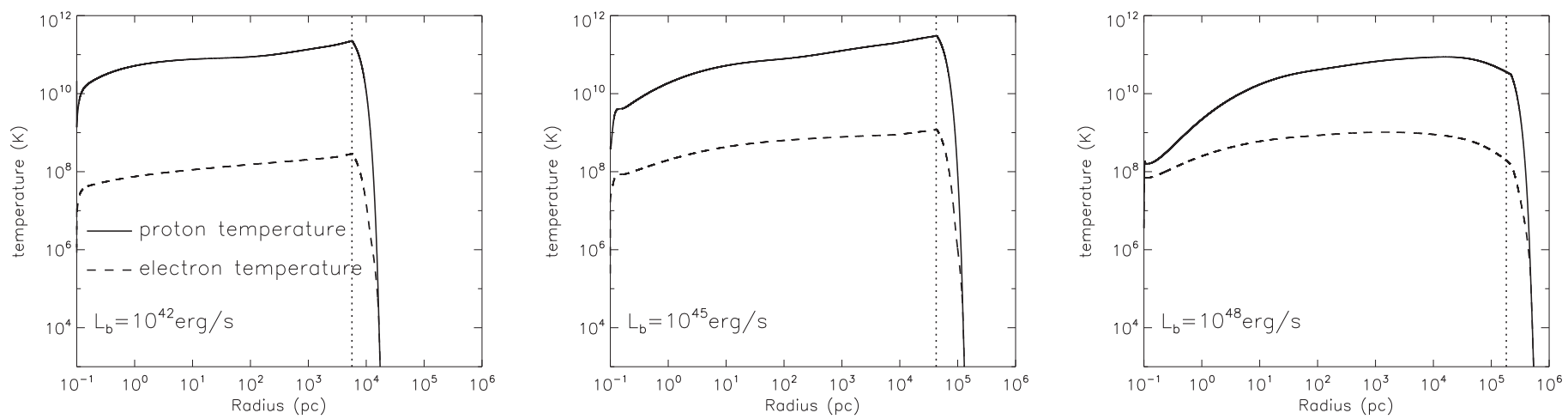

Figure 9. Evolution of proton temperature (solid curve) and electron temperature (dashed curve) in the shocked wind region for $L_{b}=10^{42}, 10^{45}$, and $10^{48}$ erg s ${ }^{-1}$, respectively. The vertical dotted lines show the Salpeter time after which the AGN shuts off.

wind is low, resulting in a relaxation time between protons and electrons (i.e., $t_{p e}$ ) shorter than the dynamic timescale.

\section{A.3. Initial Condition}

To solve Equations (8)-(12), we need to find the initial values for $v_{s}, P_{\mathrm{sw}}\left(E_{\mathrm{t}, \mathrm{sw}}\right), R_{\mathrm{rs}}$, and $v_{\mathrm{rs}}$, so that we can solve the dynamic evolution by the Runge-Kutta method. At the very early stage, the gravity is negligible and the cooling of shocked gas is not strong enough to affect the dynamics. Thus, we can assume that both the forward shock and the reverse shock are adiabatic and that the flow conserves both energy and momentum. These two conditions give the equations

$$
M_{\mathrm{sw}} v_{w}=M_{\mathrm{sg}} v_{s}+\int_{R_{\mathrm{rs}}}^{R_{s}} 4 \pi n_{\mathrm{sw}} m_{p} v_{\mathrm{sw}}(R) R^{2} d R
$$

and

$$
\begin{aligned}
\frac{1}{2} M_{\mathrm{sw}} v_{\mathrm{w}}^{2}= & E_{k, \mathrm{sw}}+E_{t, \mathrm{sw}}+E_{k, \mathrm{sg}}+E_{t, \mathrm{sg}} \\
= & \frac{1}{2} \int_{R_{\mathrm{rs}}}^{R_{s}} 4 \pi n_{\mathrm{sw}} m_{p} R^{2} v_{\mathrm{sw}}^{2} d R+\frac{3}{2} P_{\mathrm{sw}} V_{\mathrm{sw}} \\
& +\frac{1}{2} M_{\mathrm{sg}} v_{s}^{2}+\frac{1}{2} M_{\mathrm{sg}} v_{s}^{2},
\end{aligned}
$$

respectively. The integral in Equation (29) calculates the total momentum of the shocked wind, and the one in Equation (30) calculates the total kinetic energy of the shocked wind. Substituting the expressions for $M_{\mathrm{sw}}$ and $n_{\mathrm{sw}}$ into the above equations and defining $x=R_{\mathrm{rs}} / R_{s}, y=v_{s} / v_{w}$, and $\lambda=M_{\mathrm{sg}} /$ $\left(\dot{M} R_{s} / v_{w}\right)$, we can reduce the above two equations to

$$
\left(\frac{1}{y}-x\right)=\frac{4 y}{x^{2}}(1-x)+\lambda y
$$

and

$$
\frac{1}{2}\left(\frac{1}{y}-x\right)=\frac{2 y^{2}}{x^{2}}\left(\frac{1}{x}-1\right)+\frac{1}{2}\left(\frac{1}{y}-x\right)\left(1-\frac{y}{x^{2}}\right)^{2}+\lambda y^{2} .
$$

We can find the relation between $x$ and $y$ from Equation (31):

$$
y=\frac{\sqrt{x^{2}+4\left(4 \frac{1-x}{x^{2}}+\lambda\right)}-x}{2\left(4 \frac{1-x}{x^{2}}+\lambda\right)} .
$$

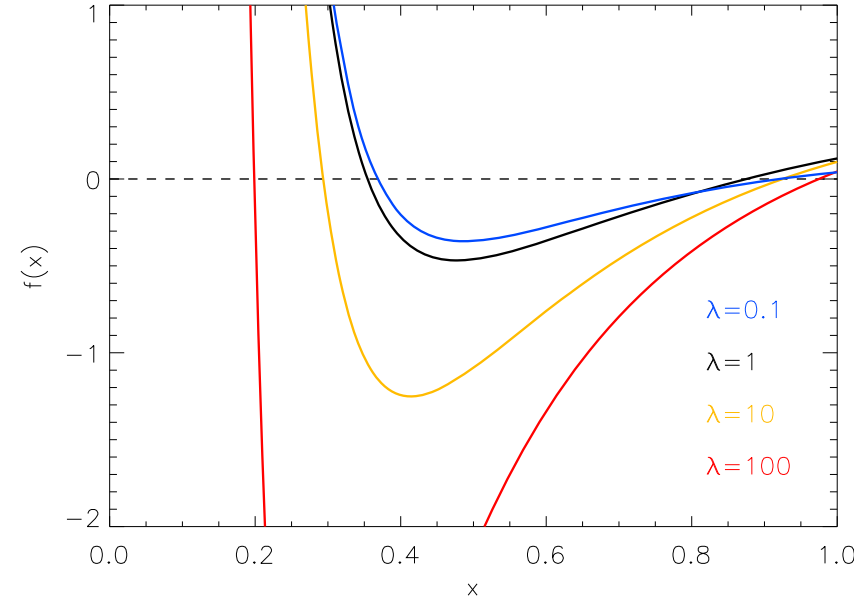

Figure 10. Value of $f$ as a function of $x$. The solutions are the values of $x$ where $f(x)=0$.

We then can solve $x$ by substituting this relation into Equation (32). Note that there is no analytic solution, but nevertheless we can solve the equation numerically by looking for the value of $x$ making the function $f \equiv \frac{2 y^{2}}{x^{2}}\left(\frac{1}{x}-1\right)+\frac{1}{2}$ $\left(\frac{1}{y}-x\right)\left(1-\frac{y}{x^{2}}\right)^{2}+\lambda y^{2}-\frac{1}{2}\left(\frac{1}{y}-x\right)=0$. Figure 10 shows the value of $f$ as a function of $x$, under different values of $\lambda$. As is shown, $x$ has two solutions for each $\lambda$. The smaller one is an extraneous root of the equation as it results in $y>1$, so only the larger one is adopted in our calculation. We select a sufficiently small radius such as $R_{s, 0}=0.1 \mathrm{pc}$ as the initial point, and we obtain the initial conditions for the dynamic evolution of the shocked ambient gas, say, $R_{\mathrm{rs}, 0}=x R_{s}$, $v_{s, 0}=x v_{w}$, and $v_{\mathrm{rs}, 0}=\left(4 v_{s, 0} / x^{2}-v_{w}\right) / 3$, as well as $P_{\mathrm{sw}, 0}$ via Equation (4).

\section{Appendix B \\ Cooling of the Accelerated Protons}

Actually, the exact solution for $N_{p}\left(E_{p}, r ; R\right)$ can be obtained by solving the energy transport equation. But this would make the overall calculation extremely time-consuming. So we here make some approximation to take into account energy losses of cosmic rays.

First, let us only consider the influences of $p p$ collisions and neglect the adiabatic cooling for the moment. Assume that a constant number fraction $f$ of the initial protons undergoes the 
interaction in a given time step. Denoting the initial number of protons at energy $E_{p}$ by $N_{0}$, we can obtain the number of protons that still have not interacted at energy $E_{p}$ after $n$ time steps by $N_{0}(1-f)^{n}$. Bearing in mind the fact that the cross section of $p p$ collision depends weakly on proton energy beyond the threshold, and assuming that a proton loses half of its energy in one $p p$ collision given the inelasticity $\kappa=0.5$, we can know that protons with initial energy $2^{i} E_{p}(i \in \mathbb{N}, i \leqslant n)$ can cool to energy $E_{p}$ after $i$ interactions. So the number of protons cooled to energy $E_{p}$ from higher energies can be given by $\sum_{i=1}^{n} N_{i} C_{n}^{i}(1-f)^{n-i} f^{i}$, where $N_{i}$ is the initial number of protons at energy $2^{i} E_{p}$ and $C_{n}^{i}=n ! / i !(n-i) !$ is the combination of taking $i$ elements without repetition from a set of total $n$ elements. For a power-law spectrum with index $\Gamma_{\mathrm{CR}}$, we have $N_{i} \simeq 2^{i\left(1-\Gamma_{\mathrm{CR}}\right)} N_{0}$. Provided a small enough time step, we can always have $(0<) f \ll 1$. The total number of protons of $E_{p}$ after $n$ time steps of injection can be given by

$$
\begin{aligned}
N & =N_{0}(1-f)^{n}+\sum_{i=1}^{n} 2^{i\left(1-\Gamma_{\mathrm{CR}}\right)} N_{0} C_{n}^{i}(1-f)^{n-i} f^{i} \\
& =N_{0} \sum_{i=0}^{n} C_{n}^{i}(1-f)^{n-i}\left(2^{1-\Gamma_{\mathrm{CR}}} f\right)^{i} \\
& =N_{0}\left[1-\left(1-2^{1-\Gamma_{\mathrm{CR}}} f\right]^{n} .\right.
\end{aligned}
$$

Defining $a \equiv\left[\left(1-2^{1-\Gamma_{\mathrm{CR}}}\right) f\right]^{-1}$, we can write the above equation as

$$
N=N_{0}\left[\left(1-\frac{1}{a}\right)^{a}\right]^{\left(1-2^{1-\Gamma \mathrm{CR}) n f} .\right.}
$$

Denoting the step size of time by $\delta t$, the fraction $f$ is the interaction rate of $p p$ collision in one step, i.e., $f=\sigma_{p p} n_{\mathrm{sg}} c \delta t$. To obtain a precise result, the time step should be as small as possible, i.e., $\delta t \rightarrow 0$, so we have $f \rightarrow 0$, and hence $a \rightarrow \infty$. Thus, we have

$$
\begin{aligned}
N & =N_{0} \lim _{a \rightarrow \infty}\left[\left(1-\frac{1}{a}\right)^{a}\right]^{\left(1-2^{\left.1-\Gamma_{\mathrm{CR} n f}\right)}\right.} \\
& =\exp \left[-\left(1-2^{\left.1-\Gamma_{\mathrm{CR}}\right)} n f\right] .\right.
\end{aligned}
$$

At a time $\Delta t$ after the initial injection, the total number of steps $n=\Delta t / \delta t$, and hence $\tau_{p p} \equiv n f=\sigma_{p p} n_{\mathrm{sg}} c \Delta t$ is the total number of collisions happening in this period. If the gas density is time dependent, as is true in this work, we have $n f=\int \sigma_{p p} n_{\mathrm{sg}} c d t$. Now considering that protons of energy $E_{p}$ are injected when the shock front is at $r$, the number of protons that have energy $E_{p}$ when the shock propagates to $R$ can be given by

$$
\begin{aligned}
N\left(E_{p}, r, R\right)= & N^{\mathrm{inj}}\left(E_{p}, r\right) \exp \\
& \times\left[-\left(1-2^{1-\Gamma_{\mathrm{CR}}}\right) \sigma_{p p}\left(E_{p}\right) c \int_{t(r)}^{t(R)} n_{\mathrm{sg}}\left(r^{\prime}\right) d t\right] \\
= & N^{\mathrm{inj}} \exp \left[-\left(1-2^{1-\Gamma_{\mathrm{CR}}}\right) \tau_{p p}\left(E_{p}, r, R\right)\right] .
\end{aligned}
$$

Now we consider the effect of adiabatic cooling and neglect the cooling via $p p$ collision for the moment. The spectrum evolution of protons injected at a certain radius $r$ can be given by the energy transport equation,

$$
\frac{\partial N}{\partial t}+\frac{\partial}{\partial E_{p}}\left(\dot{\gamma}_{a d} N\right)=0 .
$$

$\dot{E}_{p, \text { ad }} \simeq-v_{s}(r) E_{p} / r$ is the adiabatic cooling rate. Expanding the second term, we obtain

$$
\frac{\partial N}{\partial t}-\frac{v_{s}}{r} N-\frac{v_{s} E_{p}}{r} \frac{\partial N}{\partial E_{p}}=0 .
$$

We assume that the proton spectrum is a power law with index $\Gamma_{\mathrm{CR}}$, so we have $E_{p}\left(\partial N / \partial E_{p}\right)=-\Gamma_{\mathrm{CR}} N$. Then, the above equation can be written as

$$
\frac{\partial N}{\partial t}=-\left(\Gamma_{\mathrm{CR}}-1\right) \frac{v_{s}}{r} N
$$

and we find

$$
N\left(E_{p}, r, R\right)=N^{\mathrm{inj}}\left(E_{p}, r\right)\left(\frac{R}{r}\right)^{1-\Gamma_{\mathrm{CR}}} .
$$

We define $\tau_{\mathrm{ad}}(r, R) \equiv \int_{r}^{R} v d t / r^{\prime}=\int_{r}^{R} d r^{\prime} / r^{\prime}=\ln (R / r)$, so we can rewrite the above equation as $N\left(E_{p}, r, R\right)=$ $N^{\mathrm{inj}}\left(E_{p}, r\right) \exp \left[-\left(\Gamma_{\mathrm{CR}}-1\right) \tau_{\mathrm{ad}}(r, R)\right]$.

Finally, we consider both the cooling processes and have

$$
\begin{aligned}
N\left(E_{p}, r, R\right)= & N^{\mathrm{inj}}\left(E_{p}, r\right) \exp \\
& \times\left[-\left(1-2^{1-\Gamma_{\mathrm{CR}}}\right) \tau_{p p}\left(E_{p}, r, R\right)\right. \\
& \left.-\left(\Gamma_{\mathrm{CR}}-1\right) \tau_{\mathrm{ad}}(r, R)\right] .
\end{aligned}
$$

Note that in some studies only protons injected within the cooling time are considered, and these protons are assumed to be uncooled completely, i.e., $N\left(E_{p}, r, R\right)=N^{\text {inj }}\left(E_{p}, r\right) \theta\left(t_{\text {cool }}-t(r, R)\right)$, where $\theta$ is the Heaviside step function, $t_{\text {cool }}$ is the cooling time of the proton of energy $E_{p}$ and $t(r, R)$ is the time in which the shock propagates from $r$ to $R$ (e.g., Torres 2004; Lacki \& Beck 2013; Lamastra et al. 2017). Our method includes those cooled protons with $t_{\text {cool }}<t(r, R)$ and also considers cooling of those protons that are recently injected with $t_{\text {cool }}>t(r, R)$, although the difference is not significant.

\section{ORCID iDs}

Ruo-Yu Liu (i) https://orcid.org/0000-0003-1576-0961 Kohta Murase (1) https://orcid.org/0000-0002-5358-5642 Susumu Inoue (1) https://orcid.org/0000-0003-1096-9424

\section{References}

Aartsen, M. G., Abbasi, R., Abdou, Y., et al. 2013, PhRvL, 111, 021103 Aartsen, M. G., Abraham, K., Ackermann, M., et al. 2015, ApJ, 809, 98 Aartsen, M. G., Abraham, K., Ackermann, M., et al. 2016, ApJ, 833, 3 Abdo, A. A., Ackermann, M., Ajello, M., et al. 2010, ApJ, 723, 1082 Abeysekara, A. U., Albert, A., Alfaro, R., et al. 2017, ApJ, 842, 85 Abeysekara, A. U., Albert, A. M., Alfaro, R., et al. 2018, JCAP, 02, 049 Ackermann, M., Ajello, M., Albert, A., et al. 2015, ApJ, 799, 86 Ackermann, M., Ajello, M., Albert, A., et al. 2016, PhRvL, 116, 151105 Ahlers, M., \& Murase, K. 2014, PhRvD, 90, 023010

Ajello, M., Gasparrini, D., Sánchez-Conde, M., et al. 2015, ApJL, 800, L27 Andersen, K. J., Kachelriess, M., \& Semikoz, D. V. 2017, arXiv:1712.03153 Becker Tjus, J., Eichmann, B., Halzen, F., Kheirandish, A., \& Saba, S. M. 2014, PhRvD, 89, 123005

Berezinskii, V. S., \& Smirnov, A. I. 1975, Ap\&SS, 32, 461

Berezinsky, V., Gazizov, A., Kachelrieß, M., \& Ostapchenko, S. 2011, PhLB, 695, 13

Berezinsky, V., \& Kalashev, O. 2016, PhRvD, 94, 023007 
Bregman, J. N. 2007, ARA\&A, 45, 221

Buchner, J., \& Bauer, F. E. 2017, MNRAS, 465, 4348

Castor, J., McCray, R., \& Weaver, R. 1975, ApJL, 200, L107

Chang, X.-C., Liu, R.-Y., \& Wang, X.-Y. 2016, ApJ, 825, 148

Cicone, C., Maiolino, R., Sturm, E., et al. 2014, A\&A, 562, A21

Crenshaw, D. M., Kraemer, S. B., \& George, I. M. 2003, ARA\&A, 41, 117

Downes, D., \& Solomon, P. M. 1998, ApJ, 507, 615

Fabian, A. C. 2012, ARA\&A, 50, 455

Fang, K., Su, M., Linden, T., \& Murase, K. 2017, PhRvD, 96, 123007

Faucher-Giguère, C.-A., \& Quataert, E. 2012, MNRAS, 425, 605

Finke, J. D., Razzaque, S., \& Dermer, C. D. 2010, ApJ, 712, 238

Fukazawa, Y., Hiragi, K., Mizuno, M., et al. 2011, ApJ, 727, 19

Goulding, A. D., Alexander, D. M., Bauer, F. E., et al. 2012, ApJ, 755, 5

Haggard, D., Green, P. J., Anderson, S. F., et al. 2010, ApJ, 723, 1447

Halzen, F. 2017, NatPh, 13, 232

Heckman, T. M., \& Best, P. N. 2014, ARA\&A, 52, 589

Hooper, D. 2016, JCAP, 9, 002

Hopkins, P. F., Richards, G. T., \& Hernquist, L. 2007, ApJ, 654, 731

IceCube Collaboration, 2013, Sci, 342, 1

IceCube Collaboration, Aartsen, M. G., Ackermann, M., et al. 2017, arXiv:1710. 01191

Jiang, Y.-F., Ciotti, L., Ostriker, J. P., \& Spitkovsky, A. 2010, ApJ, 711, 125

Kachelrieß, M., Ostapchenko, S., \& Tomàs, R. 2012, CoPhC, 183, 1036

Kelner, S. R., Aharonian, F. A., \& Bugayov, V. V. 2006, PhRvD, 74, 034018

King, A., \& Pounds, K. 2015, ARA\&A, 53, 115

Kormendy, J., \& Ho, L. C. 2013, ARA\&A, 51, 511

Lacki, B. C., \& Beck, R. 2013, MNRAS, 430, 3171

La Franca, F., Fiore, F., Comastri, A., et al. 2005, ApJ, 635, 864

Lamastra, A., Menci, N., Fiore, F., et al. 2017, A\&A, 607, A18

Lisanti, M., Mishra-Sharma, S., Necib, L., \& Safdi, B. R. 2016, ApJ, 832, 117

Liu, R.-Y., Taylor, A. M., Wang, X.-Y., \& Aharonian, F. A. 2016, PhRvD, 94, 043008

Liu, R.-Y., Wang, X.-Y., Inoue, S., Crocker, R., \& Aharonian, F. 2014 PhRvD, 89, 083004

Liu, T., Tozzi, P., Wang, J.-X., et al. 2017, ApJS, 232, 8

Lusso, E., Comastri, A., Simmons, B. D., et al. 2012, MNRAS, 425, 623

McConnell, N. J., \& Ma, C.-P. 2013, ApJ, 764, 184
Moster, B. P., Somerville, R. S., Maulbetsch, C., et al. 2010, ApJ, 710, 903

Murase, K., Ahlers, M., \& Lacki, B. C. 2013, PhRvD, 88, 121301

Murase, K., Beacom, J. F., \& Takami, H. 2012, JCAP, 8, 030

Murase, K., Guetta, D., \& Ahlers, M. 2016, PhRvL, 116, 071101

Murase, K., Inoue, S., \& Nagataki, S. 2008, ApJL, 689, L105

Murase, K., Inoue, Y., \& Dermer, C. D. 2014, PhRvD, 90, 023007

Murase, K., \& Waxman, E. 2016, PhRvD, 94, 103006

Navarro, J. F., Frenk, C. S., \& White, S. D. M. 1996, ApJ, 462, 563

Nims, J., Quataert, E., \& Faucher-Giguère, C.-A. 2015, MNRAS, 447, 3612

Ohsuga, K., \& Mineshige, S. 2014, SSRv, 183, 353

Orr, M. R., Krennrich, F., \& Dwek, E. 2011, ApJ, 733, 77

Peng, F.-K., Wang, X.-Y., Liu, R.-Y., Tang, Q.-W., \& Wang, J.-F. 2016, ApJL, 821, L20

Peterson, B. M. 1997, An Introduction to Active Galactic Nuclei (New York: Cambridge Univ. Press)

Sazonov, S. Y., Ostriker, J. P., \& Sunyaev, R. A. 2004, MNRAS, 347, 144

Sazonov, S. Y., \& Sunyaev, R. A. 2001, AstL, 27, 481

Sherf, N., Keshet, U., \& Gurwich, I. 2017, ApJ, 847, 95

Stecker, F. W., Malkan, M. A., \& Scully, S. T. 2006, ApJ, 648, 774

Strong, A. W., \& Wolfendale, A. W. 1973, Natur, 241, 109

Tamborra, I., Ando, S., \& Murase, K. 2014, JCAP, 9, 043

Tombesi, F. 2016, AN, 337, 410

Tombesi, F., Meléndez, M., Veilleux, S., et al. 2015, Natur, 519, 436

Torres, D. F. 2004, ApJ, 617, 966

Tozzi, P., Gilli, R., Mainieri, V., et al. 2006, A\&A, 451, 457

Ueda, Y., Akiyama, M., Hasinger, G., Miyaji, T., \& Watson, M. G. 2014, ApJ, 786,104

Ueda, Y., Akiyama, M., Ohta, K., \& Miyaji, T. 2003, ApJ, 598, 886

Veilleux, S., Cecil, G., \& Bland-Hawthorn, J. 2005, ARA\&A, 43, 769

Wang, X., \& Loeb, A. 2015, MNRAS, 453, 837

Wang, X., \& Loeb, A. 2016a JCAP, 12, 012

Wang, X., \& Loeb, A. 2016b NatPh, 12, 1116

Weaver, R., McCray, R., Castor, J., Shapiro, P., \& Moore, R. 1977, ApJ, 218,377

Xiao, D., Mészáros, P., Murase, K., \& Dai, Z.-G. 2016, ApJ, 826, 133

Yu, Q., \& Tremaine, S. 2002, MNRAS, 335, 965

Yuan, C., Mészáros, P., Murase, K., \& Jeong, D. 2018, ApJ, 857, 50 